\title{
Boolean Dynamics with Random Couplings
}

\section{Maximino Aldana Susan Coppersmith Leo P. Kadanoff}

\begin{abstract}
This paper reviews a class of generic dissipative dynamical systems called $N-K$ models. In these models, the dynamics of $N$ elements, defined as Boolean variables, develop step by step, clocked by a discrete time variable. Each of the $N$ Boolean elements at a given time is given a value which depends upon $K$ elements in the previous time step.

We review the work of many authors on the behavior of the models, looking particularly at the structure and lengths of their cycles, the sizes of their basins of attraction, and the flow of information through the systems. In the limit of infinite $N$, there is a phase transition between a chaotic and an ordered phase, with a critical phase in between.

We argue that the behavior of this system depends significantly on the topology of the network connections. If the elements are placed upon a lattice with dimension $d$, the system shows correlations related to the standard percolation or directed percolation phase transition on such a lattice. On the other hand, a very different behavior is seen in the Kauffman net in which all spins are equally likely to be coupled to a given spin. In this situation, coupling loops are mostly suppressed, and the behavior of the system is much more like that of a mean field theory.

We also describe possible applications of the models to, for example, genetic networks, cell differentiation, evolution, democracy in social systems and neural networks.
\end{abstract}




\section{Introduction}

In this review, we describe the dynamics of a set of $N$ variables, or elements, which each have two possible values (say 0 and 1 ). These elements interact with each other according to some given interaction rules, specified through a set of Boolean coupling functions that determine the variables at the next time-step, and thereby give the dynamics of the system. Such a discrete stepping of a set of Boolean variables, also known in general terms as a Boolean network, is of potential interest in several different fields, ranging from gene regulation and control, to modeling democracy and social organization, to understanding the behavior of glassy materials.

The models were originally studied primarily for their biological interest, specifically by Stuart Kauffman who introduced the so-called $N-K$ model in the context of gene expression and fitness landscapes in 1969 Kauffman [1969, 1974, 1995, 1993, 1990, 1984). Since Kauffman's original work, the scientific community has found a broad spectrum of applicability of these models. Specific biological problems studied include cell differentiation (Huang and Ingber [2000|), immune response (Kauffman and Weinberger [1989|), evolution (Bornholdt and Sneppen [1998]; Zawidzki [1998]; Bornholdt and Sneppen [200d]; Ito and Gunji [1994|), regulatory networks (Bornholdt and Rohlf [2000|) and neural networks (Wang, Pichler, and Ross 1990|; Derrida, Gardner, and Zippelius [1987; Kürten [1988a|; Bornholdt and Rohlf [2000]). In the first two examples, the basic binary element might be a chemical compound, while in the last it might be the state of firing of a neuron. A computer scientist might study a similar set of models, calling the basic elements gates, and be thinking about the logic of computer design (Atlan, Fogelman-Soulie, Salomon, and Weisbuch [1981; ; Lynch [1995) or optimization (Lee and Han [1998]; Stauffer [1994]). Earlier work in the mathematical literature (Harris [1960]; Metropolis and Ulam [1953]) studied random mapping models, which are a subset of the models introduced by Kauffman. This same kind of problem has also drawn considerable attention from physicists interested in the development of chaos Glass and Hill [1998|; Luque and Solé [1998, 1997a|; Kürten and Beer [1997|; Mestl. Bagley, and Glass [1997; Bagley and Glass [1996]; Bhattacharjya and Liang [1996b]; Lynch [1995]) and also in problems associated with glassy and disordered materials (Derrida and Flyvbjerg [1986]; Derrida and Pomeau [1986; Derrida [1987b]; Derrida and Flyvbjerg [1987a ). In these examples, the Boolean element might be an atomic spin or the state of excitation of a molecule.

In some sense, the type of Boolean networks introduced by Kauffman can be considered as a prototype of generic dynamical system, as they present chaotic as well as regular behavior and many other typical structures of dynamical systems. In the thermodynamic limit $N \rightarrow \infty$, there can be "phase transitions" characterized by a critical line dividing chaotic from regular regions of state space. The study of the behavior of the system at

Version Jan. 21, 2002 ...........Edited by : Typeset on 30 October 2018 - 2h18 
and near the phase transitions, which are attained by changing the modelparameters, has been a very major concern.

As we shall describe in more detail below, these models are often studied in a version in which the couplings among the Boolean variables are picked randomly from some sort of ensemble. In fact, they are often called $N-K$ models because each of the $N$ elements composing the system, interact with exactly $K$ others (randomly chosen). In addition, their coupling functions are usually picked at random from the space of all possible functions of $K$ Boolean variables. Clearly this is a simplification of real systems as there is no particular problem which has such a generically chosen coupling. All real physical or biological problems have very specific couplings determined by the basic structure of the system in hand. However, in many cases the coupling structure of the system is very complex and completely unknown. In those cases the only option is to study the generic properties of generic couplings. One can then hope that the particular situation has as its most important properties ones which it shares with generic systems.

Another simplification is the binary nature of the variables under study. Nevertheless, many systems have important changes in behavior when "threshold" values of the dynamical variables are reached (e.g. the synapses firing potential of a neuron, or the activation potential of a given chemical reaction in a metabolic network). In those cases, even though the variables may vary continuously, the binary approach is very suitable, representing the above-below threshold state of the variables. The Boolean case is particularly favorable for the study of generic behavior. If one were to study a continuum, one would have to average the couplings over some rather complicated function space. For the Booleans, the function space is just a list of the different possible Boolean functions of Boolean variables. Since the space is enumerable, there is a very natural measure in the space. The averages needed for analytic work or simulations are direct and easy to define.

In addition to its application, the study of generic systems is of mathematical interest in and for itself.

\subsection{Structure of Models}

Any model of a Boolean net starts from $N$ elements $\left\{\sigma_{1}, \sigma_{2}, \ldots, \sigma_{N}\right\}$, each of which is a binary variable $\sigma_{i} \in\{0,1\}, i=1,2, \ldots, N$. In the time stepping, each of these Boolean elements is given by a function of the other elements. More precisely, the value of $\sigma_{i}$ at time $t+1$ is determined by the value of its $K_{i}$ controlling elements $\sigma_{j_{1}(i)}, \sigma_{j_{2}(i)}, \ldots, \sigma_{j_{K_{i}}(i)}$ at time $t$. In symbols,

$$
\sigma_{i}(t+1)=f_{i}\left(\sigma_{j_{1}(i)}(t), \sigma_{j_{2}(i)}(t), \ldots, \sigma_{j_{K_{i}}(i)}(t)\right),
$$

where $f_{i}$ is a Boolean function associated with the $i^{\text {th }}$ element that depends on $K_{i}$ arguments. To establish completely the model it is necessary to 
specify:

- the connectivity $K_{i}$ of each element, namely, how many variables will influence the value of every $\sigma_{i}$;

- the linkages (or couplings) of each element, which is the particular set of variables $\sigma_{j_{1}(i)}, \sigma_{j_{2}(i)}, \ldots, \sigma_{j_{K_{i}}(i)}$ on which the element $\sigma_{i}$ depends, and

- the evolution rule of each element, which is the Boolean function $f_{i}$ determining the value of $\sigma_{i}(t+1)$ from the values of the linkages $\sigma_{j_{1}(i)}(t), \sigma_{j_{2}(i)}(t), \ldots, \sigma_{j_{K_{i}}(i)}(t)$.

Once these quantities have been specified, equation (1.1) fully determines the dynamics of the system. In the most general case, the connectivities $K_{i}$ may vary from one element to another. However, throughout this work we will consider only the case in which the connectivity is the same for all the nodes: $K_{i}=K, i=1,2, \ldots, N$. In doing so, it is possible to talk about the connectivity $K$ of the whole system, which is an integer parameter by definition. It is worth mentioning though that when $K_{i}$ varies from one element to another, the important parameter is the mean connectivity of the system, $\langle K\rangle$, defined as

$$
\langle K\rangle=\frac{1}{N} \sum_{i=1}^{N} K_{i} .
$$

In this way, the mean connectivity might acquire non-integer values. Scalefree networks (Strogatz [2001]; Albert and Barabási [2001]), which have a very broad (power-law) distribution of $K_{i}$, can also be defined and characterized.

Of fundamental importance is the way the linkages are assigned to the elements, as the dynamics of the system both qualitatively and quantitatively depend strongly on this assignment. Throughout this paper, we distinguish between two different kinds of assignment: In a lattice assignment all the bonds are arranged on some regular lattice. For example, the $K$ control elements $\sigma_{j_{1}(i)}, \sigma_{j_{2}(i)}, \ldots, \sigma_{j_{K}(i)}$ may be picked from among the $2 d$ nearest neighbors on a $d$ dimensional hyper-cubic lattice. Alternatively, in a uniform assignment each and every element has an equal chance of appearing in this list. We shall call a Boolean system with such a uniform assignment a Kauffman net. (See Fig. 1.1.)

Of course, intermediate cases are possible, for example, one may consider systems with some linkages to far-away elements and others to neighboring elements. Small-world networks (Strogatz [2001]) are of this type.

For convenience, we will denote the whole set of Boolean elements $\left\{\sigma_{1}(t)\right.$, $\left.\sigma_{2}(t), \ldots, \sigma_{N}(t)\right\}$ by the symbol $\Sigma_{t}$ :

$$
\Sigma_{t}=\left\{\sigma_{1}(t), \sigma_{2}(t), \ldots, \sigma_{N}(t)\right\}
$$

Version Jan. 21, 2002...........Edited by : Typeset on 30 October $2018-2 \mathrm{~h} 18$ 


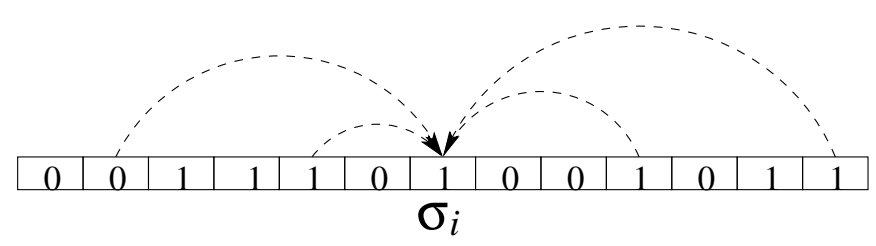

(a)

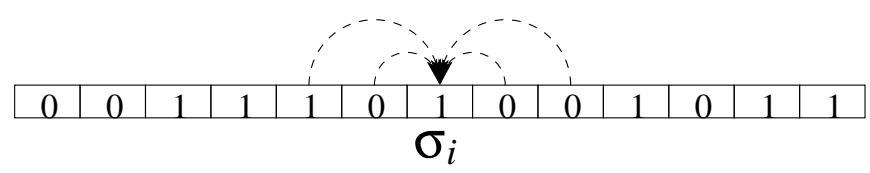

(b)

FIGURE 1.1. The different kinds of linkages in a one dimensional system. (a) In the Kauffman net the linkages of every element $\sigma_{i}$ are chosen at random among all the other elements $\sigma_{1} \ldots \sigma_{N}$. (b) In a completely ordered lattice, the linkages are chosen according to the geometry of the space. In the case illustrated in this figure, $\sigma_{i}$ is linked to its first and second nearest neighbors.

$\Sigma_{t}$ represents then the state of the system at time $t$. We can think of $\Sigma_{t}$ as an integer number which is the base-10 representation of the binary chain $\left\{\sigma_{1}(t), \sigma_{2}(t), \ldots, \sigma_{N}(t)\right\}$. Since every variable $\sigma_{i}$ has only two possible values, 0 and 1 , the number of all the possible configurations is $\Omega=2^{N}$, so that $\Sigma_{t}$ can be thought of as an integer satisfying $0 \leq \Sigma_{t}<2^{N}$. This collection of integers is the base-10 representation of the state space of the system. Although it is not essential for the understanding of the underlying dynamics of the network, this integer representation proves to be very useful in the implementation of the computational algorithms used in numerical simulations (at least for small values of $N$ ).

A note of caution is relevant at this point. We should distinguish the purely Boolean model described in this work from Kauffman's $N$ - $K$ landscape model, which provides a description of fitness landscapes by including a fitness function to be optimized. We are not going to review on fitness landscapes since abundant literature already exists on this topic (Wilke. Ronnenwinkel, and Martinetz [2001]; Kauffman [1995, 1993]). 


\begin{tabular}{|ccc|c|c|}
\hline & & Random & Canalizing \\
$\sigma_{j_{1}}$ & $\sigma_{j_{2}}$ & $\sigma_{j_{3}}$ & $f\left(\sigma_{j_{1}}, \sigma_{j_{2}}, \sigma_{j_{3}}\right)$ & $f\left(\sigma_{j_{1}}, \sigma_{j_{2}}, \sigma_{j_{3}}\right)$ \\
\hline 0 & 0 & 0 & 0 & 1 \\
0 & 0 & 1 & 1 & 1 \\
0 & 1 & 0 & 1 & 1 \\
0 & 1 & 1 & 0 & 1 \\
1 & 0 & 0 & 1 & 0 \\
1 & 0 & 1 & 0 & 1 \\
1 & 1 & 0 & 1 & 0 \\
1 & 1 & 1 & 1 & 0 \\
\hline
\end{tabular}

TABLE 1.1. Illustration of two Boolean functions of three arguments. The first function is a particular random function, whereas the second one is a canalizing function of the first argument $\sigma_{1}$. When this argument is 0 , the output of the function is always 1 , while if $\sigma_{1}=1$, the output can be either 0 or 1 .

\subsection{Coupling Functions}

The arguments of the coupling functions $f_{i}\left(\sigma_{j_{1}(i)}, \ldots, \sigma_{j_{K}(i)}\right)$ can take on $2^{K}$ different values. One specifies the functions by giving, for each of these values of the arguments, a value to the function. Therefore there are a total of

$$
\aleph=2^{2^{K}}
$$

different possible functions. In Table 1.1 we give two examples of coupling functions for the case $K=3$. There are $2^{3}=8$ configurations of the arguments $\sigma_{j_{1}(i)}, \sigma_{j_{2}(i)}, \sigma_{j_{3}(i)}$, and for each one of these configurations, the function $f_{i}$ can acquire the values 1 or 0 . For $K=3$ there are $2^{2^{3}}=256$ tables similar to the one shown in Table 1, one for each Boolean coupling function. Different tables differ in their assignments of 0's and 1's. If we assign a probability or weight to each of these functions, one gets an ensemble of possible couplings.

Possible ensenble choices abound. One ensemble used extensively by Kauffman and others is the uniform distribution in which all functions are weighted equally. Alternatively, a magnetization bias $\$$ may be applied by weighting the choice of functions with an outcome 0 with a probability $p$, and the outcome 1 with a probability $1-p$ (see, for example Bastolla and Parisi [1998b]). One may also give different weights to particular types of functions. For example, one can consider only forcing functions or canalizing functions (Stauffer [1987a]; Kauffman [1969, 1984), in which the function's value is determined when just one of its arguments is given a specific value. The second function shown in Table 1.1 is a canalizing

\footnotetext{
${ }^{1}$ The word magnetization comes from the possibility of identifying each element with an atomic spin, which is a very small magnet.
}

Version Jan. 21, 2002 ...........Edited by : Typeset on 30 October 2018 - 2h18 


\begin{tabular}{|c|c|c||c|c|}
\hline \multirow{2}{*}{$\sigma$} & \multicolumn{2}{|c||}{ Class $\mathcal{A}$} & \multicolumn{2}{c|}{ Class $\mathcal{B}$} \\
\cline { 2 - 5 } & $\mathcal{A}_{0}$ & $\mathcal{A}_{1}$ & $\mathcal{B}_{I}$ & $\mathcal{B}_{N}$ \\
\hline 0 & 0 & 1 & 0 & 1 \\
1 & 0 & 1 & 1 & 0 \\
\hline
\end{tabular}

TABLE 1.2. Boolean functions for $K=1$. The first two functions form the class $\mathcal{A}$ of constant functions, $\mathcal{A}_{0}(\sigma)=0$ and $\mathcal{A}_{1}(\sigma)=1$. The other two functions form class $\mathcal{B}$ which consist in identity $\mathcal{B}_{I}(\sigma)=\sigma$ and negation $\mathcal{B}_{N}(\sigma)=\neg \sigma$

\begin{tabular}{|c|c|c|c|c|c|c|c|c|c|c|c|c|c|c|c|c|c|}
\hline$\sigma_{j_{1}}$ & $\sigma_{j_{2}}$ & \multicolumn{2}{|c|}{ Class $\mathcal{A}$} & \multicolumn{4}{|c|}{ Class $\mathcal{B}_{1}$} & \multicolumn{8}{|c|}{ Class $\mathcal{B}_{2}$} & \multicolumn{2}{|c|}{ Class $\mathcal{C}$} \\
\hline 0 & 0 & 1 & 0 & 0 & 1 & 0 & 1 & 1 & 0 & 0 & 0 & 0 & 1 & 1 & 1 & 1 & 0 \\
\hline 0 & 1 & 1 & 0 & 0 & 1 & 1 & 0 & 0 & 1 & 0 & 0 & 1 & 0 & 1 & 1 & 0 & 1 \\
\hline 1 & 0 & 1 & 0 & 1 & 0 & 0 & 1 & 0 & 0 & 1 & 0 & 1 & 1 & 0 & 1 & 0 & 1 \\
\hline 1 & 1 & 1 & 0 & 1 & 0 & 1 & 0 & 0 & 0 & 0 & 1 & 1 & 1 & 1 & 0 & 1 & 0 \\
\hline
\end{tabular}

TABLE 1.3. Boolean functions for the case $K=2$. The 16 functions can be arranged in four different classes which differ in their symmetry properties (see text).

function. Another possibility is to specify the value of the function in order to simulate the additive properties of neurons (Bornholdt and Rohll [2000]; Genoud and Metraux [1999]; Cheng and Titterington [1994|; Wang, Pichler, and Ross [1990]; Kürten [1988a]; Derrida, Gardner, and Zippelius [1987]).

Here we enumerate some of the coupling functions occurring for different values of the connectivity $K$.

- For $K=0$ there are but two functions, corresponding to the two possible values of a Boolean variables: tautology $f=1$ and contradiction $f=0$. Together these two functions form a class which we might call $\mathcal{A}$.

- For $K=1$, in addition to the class $\mathcal{A}$, there exists another class $\mathcal{B}$ in which $f(\sigma)$ can take on the value $\sigma$, called identity, and the value $\neg \sigma$, called negation. Thus there are a total of four functions, represented as columns in Table 1.2 .

- The situation for $K=2$ has been particularly carefully studied. Here there are four classes of functions $f\left(\sigma_{1}, \sigma_{2}\right)$ Lynch [1993b; Coppersmith, Kadanoff, and Zhang [2001a $)$. Each class is invariant under making the interchange $0 \leftrightarrow 1$ in either arguments or value of $f$. The classes are $\mathcal{A}$ (two constant functions), $\mathcal{B}_{1}$ (four canalizing functions which depend on one argument), $\mathcal{B}_{2}$ (eight canalizing functions which depend on two arguments), $\mathcal{C}$, (two non-canalizing functions). These functions are explicitly shown in Table 1.3 .

Several calculations have been done by giving different weights to the Version Jan. 21, 2002...........Edited by : Typeset on 30 October $2018-2 \mathrm{~h} 18$ 
different classes (see for example Lynch [1995; Stauffer [1987a]) .

\subsection{The Updates}

Once the linkages and the $f_{i}$ 's are given, one is said to have defined a realization of the model. Given the realization, one can define a dynamics by using equation (1.1) to update all the elements at the same time. This is called a synchronous update. In this paper, we assume a synchronous update unless stated otherwise. Alternatively, one may have a serial model in which one updates only one element at a time. This element may be picked at random or by some predefined ordering scheme.

Additional choices must be made. One can:

1. Keep the same realization through all time. We then have a quenched model.

2. Pick an entirely new realization after each time step. The model is then said to be annealed [2

3. Employ a genetic algorithm in which the system slowly modifies its realization so as to approach a predefined goal (Bornholdt and Sneppen [2000]; Stern [1999]; Stauffer [1994]).

4. Intermediate choices are also possible (Baillie and Johnston [1994]; Bastolla and Parisi [1996]).

Almost always, we shall regard the real system as one which updates synchronously and which is quenched so that the interactions are fixed for all time. The annealed and sequential models will be regarded as approximations which can provide clues to the behavior of this "real system". The quenched model has time-independent dynamics describing motion within the state space of size $\Omega=2^{N}$. One iterates the model through time by using equation (1.1) and thereby obtains a dynamics for the system. Each of the $\Omega$ different initial conditions will generate a motion, which will eventually fall into a cyclical behavior.

\subsection{Symmetry Properties}

Typically, each individual realization of these models shows little or no symmetry. However, the average over realizations has quite a large symmetry group, and the symmetry is important to model behavior. For example,

\footnotetext{
${ }^{2}$ These terms have been borrowed from the physics of alloys in which something which is cooled quickly so that it cannot change its configuration is said be be quenched, and something which is held at a high temperature for a long time so that it can respond to its environment is described as annealed. Hence these terms are applied to situations in which one wants to distinguish between problems with fixed versus changing interactions.
}

Version Jan. 21, 2002 ...........Edited by : Typeset on 30 October $2018-2 \mathrm{~h} 18$ 
the random mapping model (Harris [1960]), which is the $K \rightarrow \infty$ limit of the $N-K$ model of the Kauffman net, has a full symmetry under the interchange of all states forming the state space. For finite values of $K$, the Kauffman net is symmetric under the interchange of any two basic elements. One can also have a symmetry under the interchange of the two values of each individual element if one chooses the couplings at random, or with the appropriate symmetry. One can use dynamics that have reversal symmetry (Harris [1960]; Coppersmith, Kadanoff, and Zhang [2001a, b]), and that choice will have a profound effect upon the structure of the cycles.

\subsection{Outline of Paper}

To define fully the object of study, one must describe the dynamical process and the class of realizations that one wishes to study. For example, one can fix $N$ and $K$, and study all of the properties of all realizations of frozen systems with those values. One might pick a realization at random among all possible linkages and functions, develop its properties. Then one would pick another realization, and study that. Many such steps would give us the properties of the frozen system averaged over realizations with a given $N$ and $K$. What properties might we wish to study?

In the next chapter, we describe the gross information transfer through the system by describing how the system will respond to a change in initial data or couplings. There are three different phases that have qualitatively different information transfer properties. We argue that the Kauffman net, which can transfer information from any element to any other, is qualitatively different from lattice systems, in which the information transfer occurs through a $d$-dimensional space. We argue that the information transfer on the lattice is qualitatively, and even quantitatively, similar to the kind of information flow studied in percolation problems.

Chapter 3 is concerned with the temporal recurrence in the network as reflected in the statistical properties of its cycles. Here we base our arguments upon a discussion of two important limiting cases, $K=1$, and very large $K$. The first case is dominated by situations in which there are a few short linkage loops. In the second, the Kauffman net shows a behavior which can be analyzed by comparison with a random walk through the state space. The distribution of cycle lengths is qualitatively different from any of the quantities that are commonly studied in percolation problems. So the results characterizing the cycles are substantially different from the behaviors usually studied in phase transition problems. We argue in addition that the cycles of Kauffman nets and of networks on $d$-dimensional lattices differ substantially.

Version Jan. 21, 2002............Edited by : Typeset on 30 October $2018-2 \mathrm{~h} 18$ 


\section{Information Flow}

\section{$2.1 \quad$ Response to Changes}

The first thing to study in an $N-K$ model is its response to changes. This response is important because the actual values of the elements often do not matter at all. If, for example, we pick the functions $f_{i}$ at random among the class of all Boolean functions of $K$ variables, then the ensemble is invariant under flipping the value of the $i$ th element. In that case, only changes matter, not values.

In computer studies, such changes can be followed quite simply. One follows a few different time developments of systems that are identical except for a small number of selected changes in the coupling functions or initial data, and sees how the differences between the configurations change in time. One can do this for two configurations or for many, studying pairwise differences between states, or things which remain identical across all the time-developments studied.

\subsubsection{Hamming Distance and Divergence of Orbits}

For simplicity, imagine starting out from two different possible initial states:

$$
\Sigma_{0}=\left\{\sigma_{1}(0), \sigma_{2}(0), \ldots, \sigma_{N}(0)\right\} \quad \tilde{\Sigma}_{0}=\left\{\tilde{\sigma}_{1}(0), \tilde{\sigma}_{2}(0), \ldots, \tilde{\sigma}_{N}(0)\right\}
$$

which differ in the values of a few elements. One can then observe the timedevelopment of these configurations under the same dynamics, finding, for example, the distance $D(t)$ between the configurations as a function of time

$$
D(t)=\sum_{i=1}^{N}\left(\sigma_{i}(t)-\tilde{\sigma}_{i}(t)\right)^{2} .
$$

If the transfer of information in the system is localized, this distance will never grow very large. If however, the system is sufficiently chaotic so that information may be transferred over the entire system, then in the limit of large $N$ this Hamming distance can diverge for large times.

Another interesting measure is the normalized overlap between configurations, $a(t)$, defined as

$$
a(t)=1-N^{-1} D(t) .
$$

One will wish to know whether $a$ goes to unity or a lesser value as $t \rightarrow \infty$. If the overlap always goes to unity, independently of the starting states, then the system cannot retain a nonzero fraction of the information contained in its starting configuration. Alternatively, when $a(\infty)$ is less than unity, the system "remembers" a nonzero fraction of its input data.

Version Jan. 21, 2002............Edited by : Typeset on 30 October $2018-2 \mathrm{~h} 18$ 


\subsubsection{Response to Damage}

So far, we have considered the system's response to changes of the initial data. One can also attack the quenched problem by considering two systems, each with the same initial data, but with a few of the linkages varied. Then one can ask: given such "damage" to the system, how much do the subsequent states of the system vary? Do they become more and more alike or do they diverge? What is the likelihood of such a divergence?

These considerations of robustness - both to damage and to variation in initial data - are very important for the evaluation of the effectiveness of a network, either for computations or as part of a biological system. There have been fewer studies of the effect of damage than that of initial data. Usually the two types of robustness occur together (Luque and Solé [2000]; De Sales, Martins, and Stariolo [1997|). Damage has been studied for itself (Stauffer [1994]; Corsten and Poole [1988]).

\subsection{Percolation and Phase Behavior}

\subsubsection{Percolation of Information}

In the limiting case in which $N$ approaches infinity, the different types of $N-K$ models all show three different kinds of phases, depending upon the form of information transfer in the system. If the time development transfers information to a number of elements that grows exponentially in time, the system is said to be in a chaotic phase. Typically, this behavior occurs for larger values of $K$, up to and including $K=N$. If, on the other hand, a change in the initial data typically propagates to only a finite number of other elements, the system is said to be in a frozen phase. This behavior will arise for smaller values of $K$, most especially $K=0$, and usually $K=1$. There is an intermediate situation in which information typically flows to more and more elements as time goes on, but this number increases only algebraically. This situation is described as a critical phase.

When the linkages and the hopping among configurations are sufficiently random, one can easily perform a quite precise calculation of the boundary

which separates these phases. Imagine starting with a state $\Sigma_{0}$, containing a very large number, $N$, of Boolean elements, picked at random. Imagine further another configuration $\tilde{\Sigma}_{0}$ in which the vast majority of the elements have the same value as in $\Sigma_{0}$, but nevertheless there are a large number of elements, picked at random, which are different. The Hamming distance at time zero, $D(0)$, is the number of changed elements. Now take the simplest $N-K$ system in which all the linkages and the couplings are picked at random. On average, a change in a single element will change the argument of $K$ functions, so there will be $K D(0)$ functions affected. Each of these will have a probability one half of changing their value. (The functions after all are quite random.) Thus the Hamming distance after the first time step 
will be

$$
D(1)=0.5 K D(0) .
$$

If the couplings and connections are sufficiently random, then at the start of the next step, the newly changed elements and their couplings will remain quite random. Then the same sort of equation will apply in the next time step, and the next. Just so long as the fraction of changed elements remains small, and the randomness continues, the Hamming distance will continue to change by a factor of $K / 2$ so that

$$
D(t+1)=0.5 K D(t)
$$

which then has the solution

$$
D(t)=D(0) \exp [t \ln (0.5 K)] .
$$

For $K>2$ the number of changed elements will grow exponentially, for $K<2$ it will decay exponentially, and for $K=2$ there will be neither exponential growth nor decay, and the behavior will be substantially influenced by fluctuations. Thus, by varying the value of the connectivity, the system sets down into one of the three following phases:

- Chaotic $(K>2)$, the Hamming distance grows exponentially with time.

- Frozen $(K<2)$, the Hamming distance decays exponentially with time.

- Critical $\left(K_{c}=2\right)$, the temporal evolution of the Hamming distance is determined mainly by fluctuations.

In deriving equation (2.4) we have assumed that the coupling functions $f_{i}$ of the system acquire the values 0 and 1 with the same probability $p=1 / 2$. Nonetheless, as we will see below, the chaotic, frozen and critical phases are also present in the more general case in which the coupling functions $f_{i}$ evaluate to 0 and 1 with probabilities $p$ and $1-p$ respectively. For a given value of $p$, there is a critical value $K_{c}(p)$ of the connectivity below which the system is in the frozen phase and above which the chaotic phase is attained. Conversely, for a given connectivity $K \geq 2$, a critical value $p_{c}(K)$ of the probability bias separates the chaotic and the frozen phases.

The behavior of the normalized Hamming distance, $D(t) / N$, can be seen in figures 2.1 and 2.2, which respectively are for the Kauffman net and a two-dimensional lattice system. In both cases the system has $N=10^{4}$ elements and the connectivity is $K=4$. The linkages of every element of the Kauffman net are chosen randomly, whereas in the two-dimensional lattice each element receives inputs from its four nearest neighbors. Both

Version Jan. 21, 2002 ...........Edited by : Typeset on 30 October $2018-2 \mathrm{~h} 18$ 

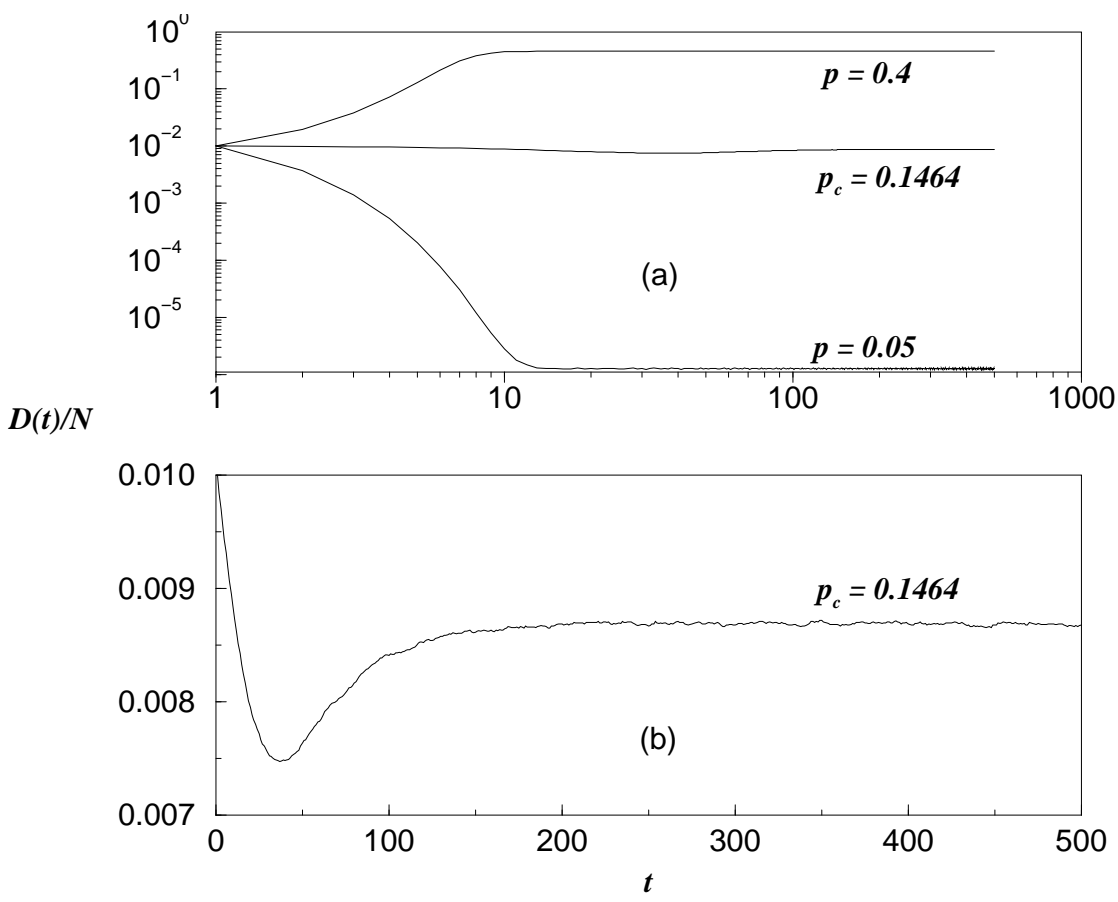

FIGURE 2.1. Hamming distance as a function of time for a Kauffman net composed of $N=10000$ elements and connectivity $K=4$. (a) Log-log graph showing the Hamming distance for the three different regimes of the system: frozen $(p=0.05)$, critical $\left(p_{c}=(1-\sqrt{1 / 2}) / 2 \simeq 0.1464\right)$ and chaotic $(p=0.4)$. In all the cases the initial Hamming distance was $D(0)=100$. (b) Hamming distance for the critical phase $\left(p=p_{c}\right)$ but in a non-logarithmic graph. Note that the Hamming distance initially decreases, and then it rises again to saturate at a constant value that depends weakly on system size.

figures contain three curves, with the parameter $p$ picked to put the systems into the three different phases. For the Kauffman net $p_{c}=(1-\sqrt{1 / 2}) / 2$ (see equation (2.8) below). The value of $p_{c}$ is not very well known for the two-dimensional lattice, but the best numerical estimations indicate that $p_{c} \simeq 0.29$ for an infinite lattice (Stauffer [1988); Stölzle [1988); Weisbuch and Stauffer [1987]; Stauffer [1987b]; Derrida and Stauffer [1986]). For finite lattices the value of $p_{c}$ has been defined as the average over realizations of the value of $p$ at which a first cluster spanning the whole net appears (Lam [1988]). For a $100 \times 100$ lattice this value is $p_{c} \simeq 0.27$.

In the frozen phase the distance has an initial transient but then quite quickly approaches an asymptotic value. In the chaotic phase the distance shows an exponential rise followed by a similar saturation. These behaviors are almost the same for both the Kauffman net and the two-dimensional lattice. On the other hand, in the critical phase the behavior of the HamVersion Jan. 21, 2002 ...........Edited by : Typeset on 30 October $2018-2 \mathrm{~h} 18$ 


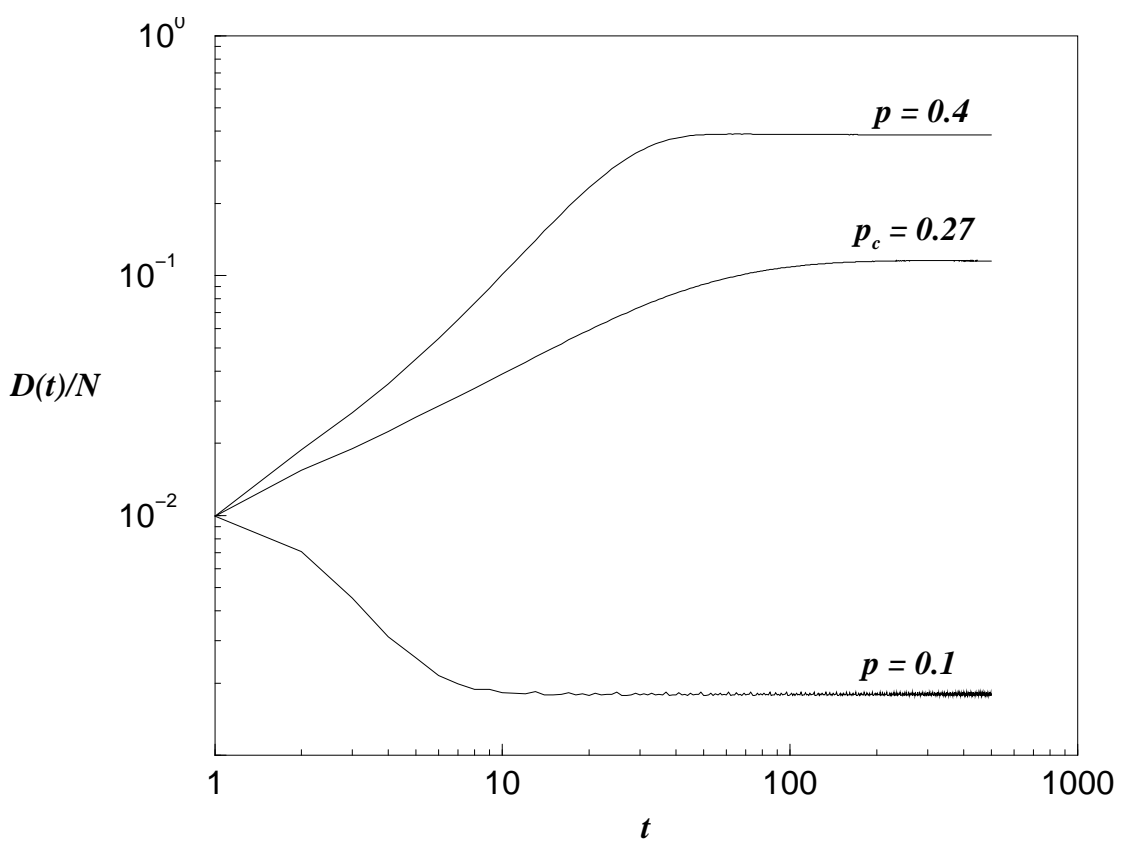

FIGURE 2.2. Hamming distance in a two-dimensional lattice composed of $N=100 \times 100$ elements. Every node in the lattice receive inputs from its four first nearest neighbors $(K=4)$. The three curves (in log-log scale) show the behavior of the Hamming distance in the three regimes: frozen $(p=0.1)$, critical $\left(p_{c}=0.27\right)$ and chaotic $(p=0.4)$. Note that in the critical phase the Hamming distance initially increases algebraically and then saturates at a constant value that depends on system size.

ming distance is very different in these two systems. In the Kauffman net the distance initially decreases and then increases again, asymptotically approaching a constant value that depends weakly on system size. In contrast, for the lattice the Hamming distance initially grows and then saturates. We will see later that, within the framework of the annealed approximation, the normalized Hamming distance for an infinite Kauffman net approaches zero monotonically in both the frozen and the critical phases (exponentially in the frozen phase, and algebraically in the critical phase). As far as we can tell, the non-monotonic behavior of the Hamming distance in finite systems at $K_{c}$ shown in Fig. 2.1b has not yet been explained.

\subsubsection{Limitations on the mean field calculation}

Let us look back at the argument which led to equation (2.4). Calculations like this, in which actual system properties are replaced by average system properties are in general called "mean field" calculations.

Naturally, the results derived as equation (2.4) depend crucially upon the

Version Jan. 21, 2002...........Edited by : Typeset on 30 October $2018-2 \mathrm{~h} 18$ 
assumptions made. The derivation follows from the assumption that the $f_{i}$ 's in each step are effectively random. (See also the derivations of equations (2.6) and (2.7) below, which also depend upon the randomness assumption.) The randomness will certainly be correct in the annealed situation, in which the couplings are reshuffled in each step. It will also be true in Kauffman net in the limiting situation in which $K=\infty$. In that case, information is spread out over the entire system and thus has a very small chance of correlation among the $f_{i}$ 's. The Kauffman net has a likely configuration that permits the replacement of the actual values of the $f_{i}$ 's by their statistical distribution (Hilhorst and Nijmeijer [1987]). However, the approximations used here will not always work. Specifically, they fail in all kinds of finite $N$ situations, or in situations in which the linkages are arranged in a finitedimensional lattice. In that case, the assumed randomness of the $f_{i}$ does not hold, because their arguments are not random, and the derived equations will not work. To see this in the simplest example choose $K=N=1$ with quenched couplings. A brief calculation shows that for any one of the four possible $f_{i}$ 's, after a one-step initial transient $a(t+2)=a(t)$. That does not agree with equation (2.6) derived below. In fact, for any finite dimension and linkages which involve short-range couplings, the overlap is not unity at long times even in the frozen phase. More generally, if the system is put onto a finite dimensional lattice, or if the functions are not picked at random, or if the initial elements are not random, couplings initially used can be correlated with couplings used later on. Then the information transfer will be different and equation (2.4) will fail.

However, the principle that there can be a phase transition in the kind of information flow remains quite true for a $d$-dimensional lattice, and for other ensembles of coupling functions.

\subsubsection{Connections to percolation problems}

The transfer of information just described is quite similar to the transfer which occurs in a typical phase transition problem. Generically, these problems have three phases: ordered, critical, and disordered (Ma [1976); Kadanoff [2000). The bare bones of such an information transfer problem is described as a percolation problem (Stauffer [1985]).

In one sort of percolation problem one considers a lattice. Each bond or link of the lattice is picked to be either connected or disconnected. The choice is made at random and the probability of connecting a given link is picked to be $q$. Now one asks about the size of the structure obtained by considering sets of links all connected with one another. For small values of $q$, these sets tend to be small and isolated. As $q$ is increased, the clusters tend to get larger. At sufficiently large values of $q$, one or more connected clusters may span the entire lattice. There is a critical value of the probability, denoted as $q_{c}$, at which such a spanning cluster just begins to form. Properties of the large clusters formed near that critical point

Version Jan. 21, 2002 ...........Edited by : Typeset on 30 October $2018-2 \mathrm{~h} 18$ 
have been studied extensively (Stauffer [1985). The resulting behavior is "universal" in that for an isotropic situation, the critical properties depend only upon the dimensionality $d$ of the lattice, at least when $d$ is less than four. For $d>4$, the percolating system obeys mean field equations. When the symmetry properties change, the critical behavior does change. For example, a system with directed percolation (Stauffer [1985); Owezarek, Rechnitzer, and Guttmann [1997]) has the information flow move preferentially in a particular direction. The critical behavior of directed percolation is different from that of ordinary percolation. It is attractive to explore the connection between the phase transition for percolation, and the one for the $N-K$ model.

Several authors have constructed this connection in detail. For example, Hansen [1988a] looked at the $N-K$ model on lattices for 2,3 and 4 dimensions and demonstrated numerically that the phase transition occurred when the information transition probability reached the critical value for percolation on the corresponding lattice. Stölzle [1988] showed the connection to percolation for both sequential and parallel updating for a two dimensional lattice. However, Hansen [1988b] took very special forms of the connections, using only rules with connectivities of the form of a logical "or". This did not result in behavior like simple percolation but instead quite a different phase transition problem, related to the behavior of diodes and resistors. At roughly the same time, Stauffer [1988 indicated a close numerical correspondence to the two dimensional percolation problem both in $p_{c}$ and also in the fractal dimension of the spanning cluster. (For $p_{c}$ see also Lam [1988].) He also defined an order parameter, essentially a Hamming distance, that, when plotted as a function of $\left(p-p_{c}\right)$, looked just like a typical critical phenomena result. He argued that the $N-K$ model is equivalent to directed percolation. More specifically, Obukhov and Stauffer [1989] argued that the quenched problem has a critical point which is in the same "universality class" (Kadanoff [2000) as directed percolation. This would imply that the critical point is essentially the same as that of the directed percolation problem. The qualitative properties of both the ordered and the frozen phases would also be essentially similar in the percolation case and the $N-K$ lattice. In the latter situation, the preferred direction would be that of the "time" axis. The structure of both would vary with dimensionality and become like that of mean field theory above four dimensions. This is the same mean field theory which describes the Kauffman net. Thus, the behavior of information transfer in $N-K$ problems was mostly understood in terms of percolation.

\subsection{Lattice versus Kauffman net}

We can now point to an important difference between systems in which all elements are coupled to all others, as in the Kauffman net, and lattice systems in which the elements which are "close" to one another are likely

Version Jan. 21, 2002...........Edited by : Typeset on 30 October $2018-2 \mathrm{~h} 18$ 
to be mutually coupled. "Closeness" is a reciprocal relation. If $a$ is close to $b$, then $b$ is also close to $a$. Therefore, close elements are likely to be coupled to one another and thereby form a closed linkage loop. Any large$N$ lattice system might be expected to form many such loops. When $K$ is small, different spatial regions tend to be unconnected and so many different modules will form 3 . The dynamics of the elements in different modules are independent. In contrast, in a Kauffman net, influence is not a reciprocal relation. If element $\sigma_{j}$ appears in the coupling function $f_{i}$ associated with element $\sigma_{i}$, there is only a small chance, proportional to $(K / N)$, that $\sigma_{i}$ will appear in $f_{j}$. For large $N$ and small $K$, the probability that a given element will participate in a linkage loop will be quite small, so there will then be a small number of modules. When $K$ is small, the number of modules in uniformly coupled systems grows more slowly than the system size, while in lattice systems the number of modules is proportional to the size of the system. This distinction will make a large difference in the cycle structure. For the flow of information the difference between the two kinds of nets is more quantitative than qualitative. One can see the similarity between them by comparing the curves shown in figures 2.1 and 2.2 .

\subsection{Calculations of overlap and divergence}

Before coming to a careful description of the phases, we should describe more fully the kinds of calculation of overlap that can be performed. Equation (2.4) is just the beginning of what can be done with the trajectories of states in this problem. In fact, exactly the same logic which leads to that equation can give a much more general result. If the overlap between two states at time $t$ is $a(t)$, and if the elements which are different arise at random, then the probability that the arguments of the function $f_{i}$ will be the same for the two configurations is

$$
\rho=[a(t)]^{K} .
$$

If all arguments are the same, then the contribution to the overlap at time $t+1$ is $1 / N$. (The $N$ arises from the normalization of the overlap.) If one or more arguments of the coupling function are different in the two configurations, and the functions $f_{i}$ are picked at random, then the chance

\footnotetext{
${ }^{3} \mathrm{~A}$ module is a loop or more complex topology of dependencies in which all the functions are non-constant, plus all other elements that are influenced by that structure. See section 3.1.

${ }^{4}$ The interested reader will recall that in quantum field theory and statistical mechanics, mean field theory appears in a situation in which fluctuations are relatively unimportant. This will arise when the analog of linkage loops make a small contribution. Then, the physicist puts the effect of loops back into the problem by doing what is called a loop expansion. Since they both expand in loops, the percolation mean field theory and the mean field theory of the Kauffman net are essentially the same.
}

Version Jan. 21, 2002...........Edited by : Typeset on 30 October $2018-2 \mathrm{~h} 18$ 
of having the same functional output is $1 / 2$ and the contribution to the overlap is $1 /(2 N)$. Since there are $N$ of such contributions, weighted by $\rho$ and $1-\rho$ respectively, the equation for the overlap defined by equation (2.3) is

$$
a(t+1)=\left[1+[a(t)]^{K}\right] / 2 .
$$

There are several possible variants of this equation. For example, if the different outcomes 0 and 1 of the function $f_{i}$ are weighted with probabilities $p$ and $1-p$ respectively, to produce a sort of magnetization bias, then equation (2.6) is replaced by (Derrida and Pomeau [1986); Derrida and Stauffer [1986])

$$
a(t+1)=1-\left[1-[a(t)]^{K}\right] / K_{c},
$$

where $K_{c}$ is given in terms of $p$ as

$$
K_{c}=1 /[2 p(1-p)]
$$

In the limit $t \rightarrow \infty, a(t)$ asymptotically approaches the fixed point $a^{*}$, which obeys, from equation (2.7)

$$
a^{*}=1-\left[1-\left[a^{*}\right]^{K}\right] / K_{c} .
$$

We might expect equation (2.9) to reflect the three-phase structure of the problem, and indeed it does. Fig. 2.3a shows the graph of the mapping $F(a)=1-\left[1-a^{K}\right] / K_{c}$ for different values of $K$, and Fig. 2.3b shows the bifurcation diagram of equation (2.9). Both graphs were calculated with $p$ chosen so that $K_{c}=3$. As can be seen, if $K \leq K_{c}$ there is only one fixed point $a^{*}=1$, whereas for $K>K_{c}$ the fixed point $a^{*}=1$ becomes unstable as another stable fixed point, $a_{s}^{*} \neq 1$, appears. The value of the infinite time overlap $a^{*}$ describes the fraction of elements whose value is sensitive to the cycle entered.

When $K>K_{c}$, the system is chaotic; $a^{*}$ is less than one even when the starting points are quite close to one another. This reflects the fact that the system has a long-term behavior which can include some cyclic oscillations, the initial data determining the cycle entered. (We discuss the cycles in detail in the next chapter.) As $K$ approaches $K_{c}$ from above, $a^{*}$ increases since fewer elements have final values which are sensitive to the initial data. On the other hand, for $K \leq K_{c}$, the infinite time overlap is exactly one and therefore the proportion of elements whose final value depends upon the starting point is precisely zero. Thus, independently of the starting point, the system is always stuck in essentially the same configuration. This surprising result pertains to the Kauffman net. In contrast, for any lattice system with $K>0$ the final overlap is less than unity, reflecting the fact that the system can store information about initial data in some finite proportion of its elements. Such storage is impossible for the Kauffman net.

Version Jan. 21, 2002 ...........Edited by : Typeset on 30 October $2018-2 \mathrm{~h} 18$ 

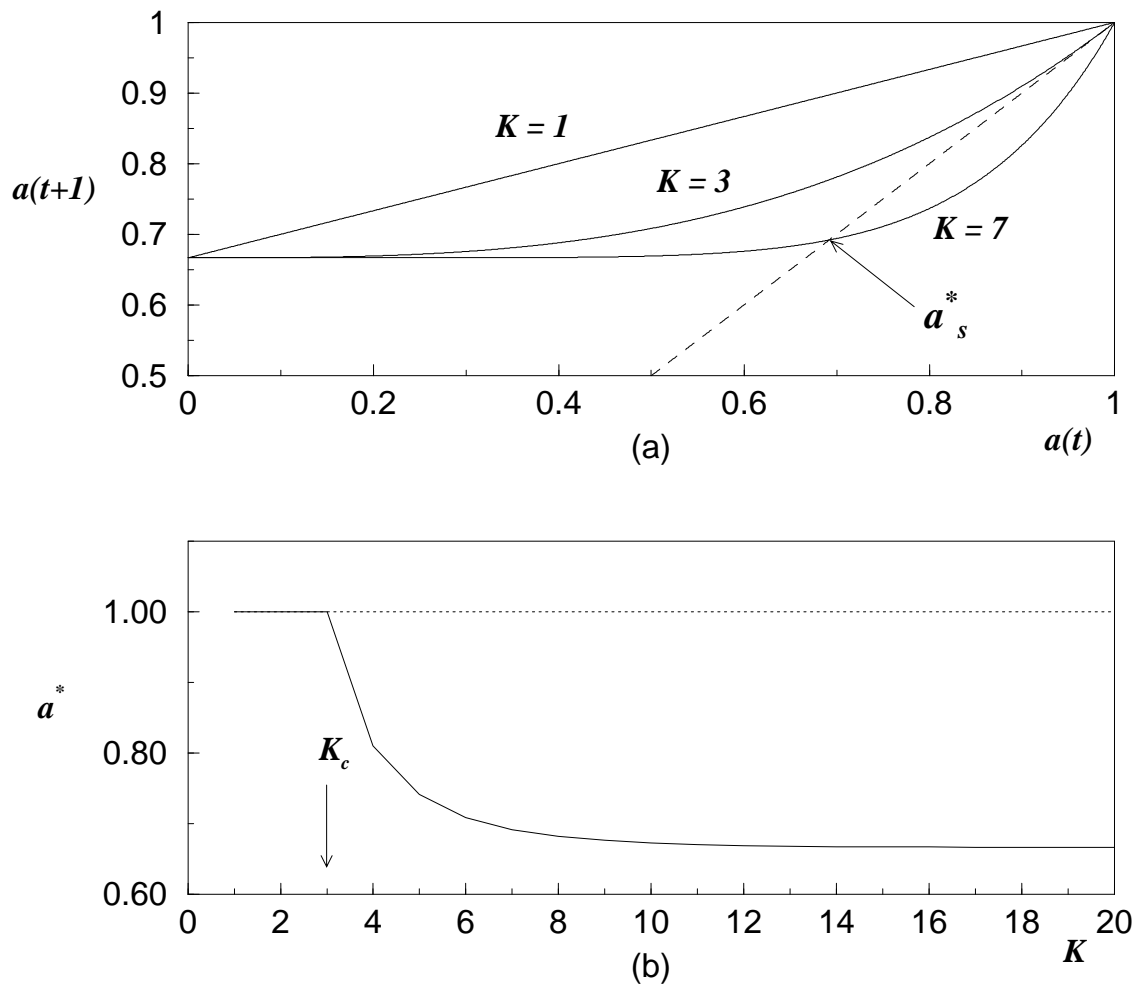

FIGURE 2.3. (a) The mapping $F(a)=1-\left[1-a^{K}\right] / K_{c}$ (see Eq. (2.7)) for $K_{c}=3$ and three different values of $K$ (solid curves), corresponding to the three different phases of the system. The dotted line is the identity mapping. (b) Bifurcation diagram of equation (2.9). For $K \leq K_{c}$ the only fixed point is $a^{*}=1$. For $K>K_{c}$ the previous fixed point becomes unstable and another stable fixed point $a_{s}^{*}$ appears.

It is worth noticing that for a given value of $K$, the system can be put in one of the three different phases by adjusting the value of the probability bias $p$. The critical value of $p$ is then obtained by inverting equation (2.8), which gives the critical line separating the frozen phase from the chaotic phase in the $p-K$ parameter space, as illustrated in Fig. 2.4.

The rigidity of the Kauffman net was emphasized by Flyvbjerg (Flyvbjerg [1988, 1989]), who wished to establish the equation for the stable core, the set of variables whose values at time infinity do not depend upon initial data (see also Lynch [1995, 1993a. b]; Bastolla and Parisi [1998b].) He calculated the time dependence of the proportion of variables which had already settled down, and thereby found a closed form for the size of the core. He found that in the ordered state of the Kauffman net the fraction of elements in the core is unity. Another kind of rigidity is studied Version Jan. 21, 2002...........Edited by : Typeset on 30 October $2018-2 \mathrm{~h} 18$ 


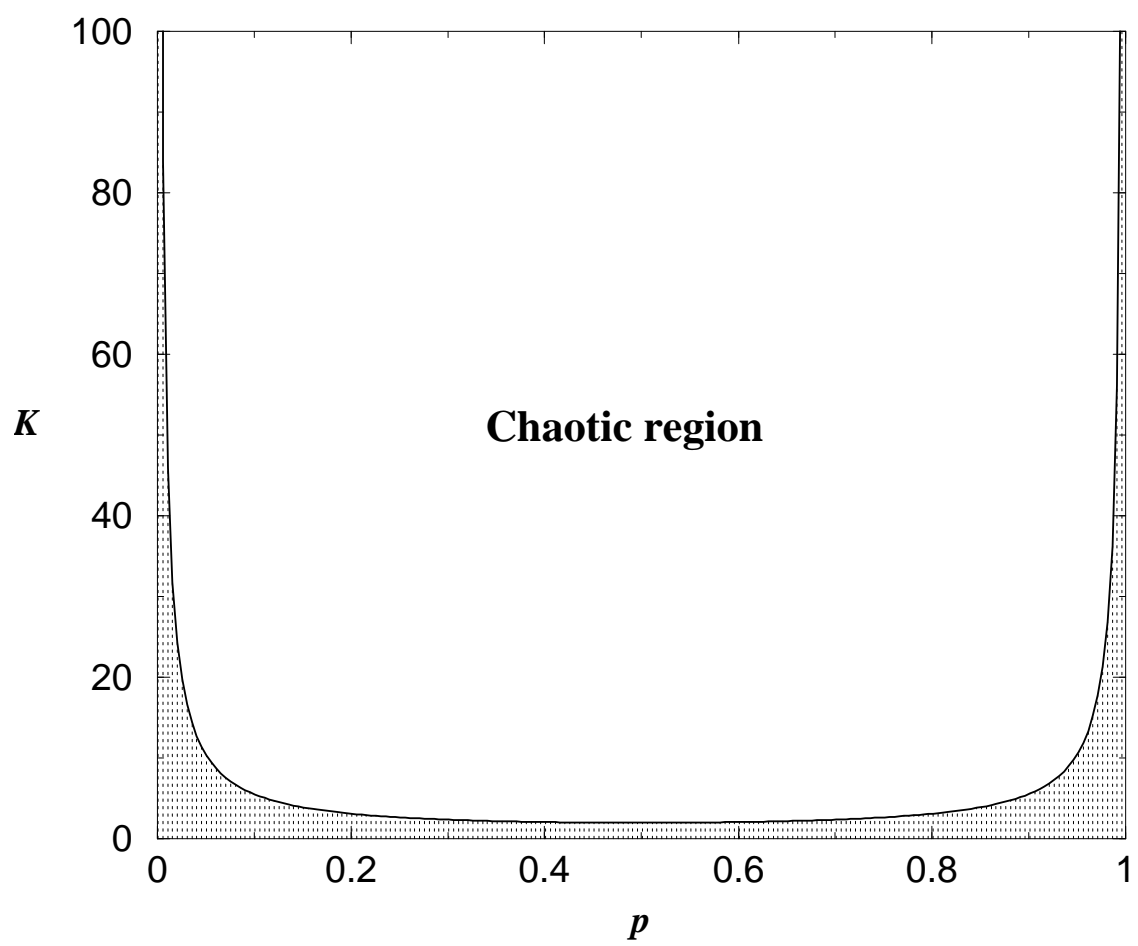

FIGURE 2.4. Phase diagram for the $N-K$ model. The shaded area corresponds to the frozen phase, whereas the upper region corresponds to the chaotic phase. The curve separating both regions is the critical phase $K_{c}=[2 p(1-p)]^{-1}$.

by counting the weak elements. In a particular realization, elements are termed weak if changing their value never affects the long-term behavior of the system. Many authors have studied these elements (see for example Lynch [1995, 1993a, b]). In the Kauffman net, but not in the lattice system, the proportion of weak elements is unity throughout the frozen phase. 


\section{Cycle Behavior}

For quenched systems the evolution functions $f_{i}$ are independent of time. For any initial condition, the system eventually returns to a previously visited point and then cycles repeatedly. The state space, which consists of the $\Omega=2^{N}$ configurations of the system, breaks up into different cycles and their basins of attraction, as shown schematically in Fig. 3.1. Each initial condition will eventually fall in one of these cycles, which are determined by the evolution functions and the linkages.

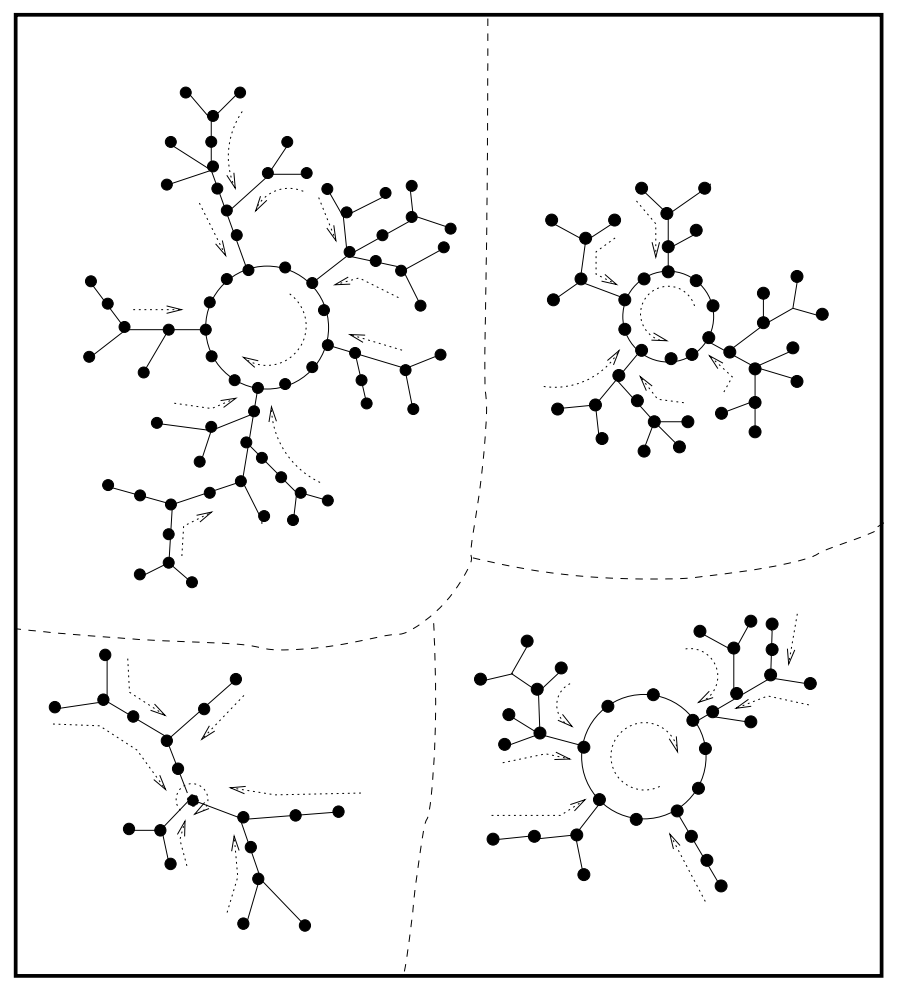

FIGURE 3.1. Schematic representation of the state space of the $N-K$ model. Each state is represented as a bold point. Under the quenched dynamics, the state space is broken down into several cycles, or attractors, represented as circles. Each initial state eventually will end up in one of these cycles (the arrows show the direction of the flow). The totality of states which evolve towards a given cycle, is the basin of attraction of that cycle. Note that there can be attractors consisting of only one point, corresponding to a cycle of period 1 .

The description of a cycle is, in some sense, much more complex than the description of orbit separation. In separation, one is dealing with a very gross property of the system: Different parts of it can behave independently 
and additively in the separation process. We utilized this fact in our calculations of overlaps and Hamming distances carried out in sections 2.2.1 and 2.4 above. On the other hand, to get a cycle to close, each and every element must simultaneously return to its value at a previous time-step. This is a much more delicate process and makes for much more delicate calculations. As we saw, information flow in $N-K$ systems is closely analogous with the well-studied behavior of percolation problems. In contrast, the behavior of cycles in $N-K$ models is something special, not closely analogous to any of the usual calculations for the usual systems of statistical mechanics. When the $N-K$ system forms cycles, one can ask many quantitative questions. One might wish to know the number of steps the system takes before it falls into a cycle (called the transient time), and about the length of the cycle. For each cycle, there is a basin of attraction, which is the set of initial states which eventually fall into that cycle. Therefore, one can develop a quantitative description of the distribution of cycle lengths, transient lengths, basin sizes, etc. either within a given realization or averaged over realizations.

\subsection{Linkage Loops and Cycles}

The structure of the linkages is an important ingredient in determining the nature of the cycles in the system. Each element can appear in the coupling functions for other elements. These in turn can appear in the couplings of others. For each element one can trace out its descendants, i.e., the elements it affects. Similarly, one can chain backward and find the ancestors for each element, i.e., the elements which affect it. For a cycle of length longer than one to exist, at least one element must be its own ancestor, and thus its own descendant. (If no such element existed, one could trace back and find elements with no ancestors. They would then have fixed values and be stable elements. The elements controlled by only them would also be stable. The line of argument would go forward until it was found that all elements were stable.) Fig. 3.2 illustrates the idea of ancestors and descendants for the case $K=1$. As can be seen, the connections between different elements of the network give rise to linkage loops and linkage trees, each tree being rooted in a loop. Flyvbjerg and Kjaer [1988] stress the importance of elements which are their own ancestors by pointing out that any unstable element must be influenced by at least one such element. Of particular relevance are those elements which belong to a loop in which there are only non-constant functions. These self-influencing elements, and the unstable elements that they influence, are called the relevant elements of the system. They naturally form themselves into groups called modules (Thieffry and Romero [1999]; Bastolla and Parisi [1998a; Zawidzki [1998]). Different modules do not influence one another and fully determine the cycle structure. Notice that the chain of linkages by which an element can influence itself, namely its linkage loop, is completely defined by the linkages, i.e.

Version Jan. 21, 2002...........Edited by : Typeset on 30 October $2018-2 \mathrm{~h} 18$ 
(a)

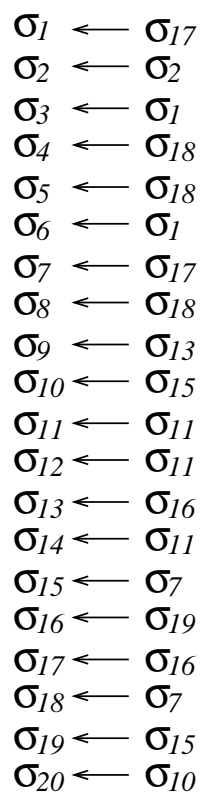

(b)
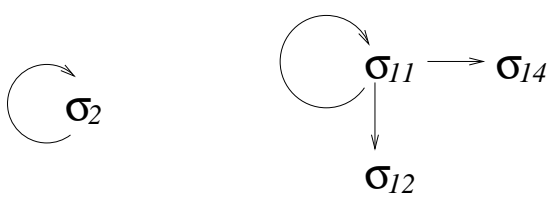

FIGURE 3.2. Linkage loops for an $N-K$ net of $N=20$ elements and connectivity $K=1$. (a) Particular realization of linkage assignment in the net. The first column is the list of the $N$ elements of the net, $\left\{\sigma_{1}, \sigma_{2}, \ldots, \sigma_{N}\right\}$, and the second column shows the particular linkage every element has been assigned. (b) Schematic representation of the linkage loops. Each arrow points towards the descendants of a given element. In this particular realization there are three modules, one of which consits of only one element, $\sigma_{2}$.

by the specific assignment of the different elements in the $f_{i}$ 's. Linkage loops do not describe the functions themselves. Consequently, such loops are necessary but not sufficient for the existence of non-trivial cycles. Only loops formed by relevant elements (with non-constant functions) are important in determining the properties of the limit cycles of the network. The number of such cycles and their lengths depend crucially on the modular organization of the relevant variables (Flyvbjerg and Kjaer [1988); Bastolla and Parisi [1998b, al). Consequently, linkage loops and relevant elements will form an important part of our further discussions.

\subsection{Phase Transitions for Cycles.}

In the previous chapter, we saw that $N-K$ systems fall into different phases, depending upon how effectively they transfer information. The cycles can be quite different in the different phases. The chaotic phase is characterized 
by very long cycles, in which the typical cycle-lengths grow as a power of the size, $\Omega=2^{N}$, of the entire state space. Each cycle includes the participation of many different elements which undergo a strongly correlated dynamics. In this phase, the transients are similarly long and complex. In contrast, the frozen phase tends to have much shorter and simpler cycles, and also shorter transients in which the individual modules do not grow as the system gets larger. If the system sits on a lattice of low dimension, the different modules are spatially localized. Each module shows a strong correlation in its dynamics, but different modules move independently. The critical phase has larger modules and has a behavior intermediate between the frozen and the chaotic phase. These behaviors are known in considerable detail.

The remainder of this chapter describes in detail the behaviors of the different phases. We start by discussing the exactly solvable models which give a solid description of the limiting behavior of the phases. We then describe how information obtained from simulations and the exact solutions can be pieced together to give a qualitative description of the phases.

\subsection{Soluble Models.}

\subsubsection{Independent Subsystems}

Here we do a preliminary calculation which will be of use in interpreting results involving cycles. Imagine a system composed of $N$ independent subsystems. Each subsystem has a probability $\rho_{l}$ of having a cycle of length $l$. We imagine that $\rho_{l}$ gets quite small for large $l$ and ask what is the chance of finding a long cycle in the entire system. Notice that the chance of not finding a piece with a cycle of length $l$ in the entire system is

$$
q_{l}=\left(1-\rho_{l}\right)^{N} \approx \exp \left(-N \rho_{l}\right) .
$$

If then, $\rho_{l}$ varies exponentially with $l$ (the justification for this assumption will be given below), namely, if

$$
\rho_{l}=A \exp (-\alpha l),
$$

then we might expect to find parts with all cycle lengths up to

$$
l_{m x}=(\ln N) / \alpha
$$

(so that $q_{l}$ is not that small, say of order $q_{l} \sim e^{-A}$ ). To make a long cycle in the entire system, one puts together many sub-cycles of different lengths, $l_{i}, i=1,2, \ldots, N$. The total cycle length, $L$, is the smallest number divisible by each of the $l_{i}$ 's. Then $L$ will be a product of all prime numbers, $p_{r}$, which are less than $l_{m x}$, each raised to a power $s_{r}$ which is the largest integer for which the inequality

$$
\left[\frac{l_{m x}}{p_{r}^{s_{r}}}\right] \geq 1
$$

Version Jan. 21, 2002............Edited by : Typeset on 30 October $2018-2 \mathrm{~h} 18$ 
is satisfied ( $[x]$ being the integer part of $x)$. Hence, to a decent approximation, the largest cycle length $L_{m x}$ will be

$$
L_{m x} \approx\left(l_{m x}\right)^{\pi\left(l_{m x}\right)},
$$

where $\pi(l)$ is the number of primes less than $l$, which can be estimated in the asymptotic limit of large $l$ as 5

$$
\pi(l)=l / \ln l .
$$

In the end then, the longest cycle length $L_{m x}$ obeys

$$
\ln L_{m x} \approx \pi\left(l_{m x}\right)\left(\ln l_{m x}\right) \approx l_{m x},
$$

so that

$$
L_{m x} \approx N^{1 / \alpha} .
$$

We have reach the remarkable conclusion that even though the probability of long cycles in each component of the system falls exponentially, the typical maximum cycle length in the entire system depends algebraically upon the size of the system. This calculation does not apply directly to Kauffman nets because we have not accounted for the fact that the different modules have different distributions $\rho_{l}$, but nonetheless it is instructive.

\subsection{2 $K=0$}

The case in which $K$ is zero is simple and uninteresting. After the first step, each element has a value which is completely determined by its function $f_{i}$. Each element remains with the value it had at time one for all subsequent times. Thus the system is completely frozen.

\subsection{3 $K=1$}

In an important paper, Flyvbjerg and Kjaer [1988] analyze the structure of the case in which each coupling function depends upon the value of just one element. To analyze this case the authors focus upon those spins which are ancestors of themselves. As we have seen (see Fig. 3.2), each such element forms part of a coupling loop of a length which we will denote as $m$. No information may pass into such a loop from other parts of the system. Each element in the loop may affect others, but the affected elements either are constant or they inherit the cycle length of the coupling loop. In a lattice system, each element is coupled with a neighboring element. In any finite number of dimensions, there is a nonzero probability that two nearest

\footnotetext{
${ }^{5} \mathrm{~A}$ better approximation is $\pi(l)=1 /(\ln l-1)$ Rosser and Schoenfeld [1962). Also, in this reference it is shown that $l / \ln l<\pi(l)<1.0423 l / \ln l$ for all $l>10$.
}

Version Jan. 21, 2002 ...........Edited by : Typeset on 30 October $2018-2 \mathrm{~h} 18$ 
neighbor sites are inputs to each other. Therefore, a system with $N$ sites will contain a number of loops that is proportional to $N$. In contrast, on the Kauffman net, the couplings are not to neighbors but randomly chosen from the whole system. When $K=1$, the probability that two sites are inputs to each other is proportional to $1 / N$, and the average number of loops grows logarithmically with $N$ (Bastolla and Parisi [1998a ). Flyvbjerg and Kjaer calculate the probability of observing $n_{m}$ loops of length $m$ in a system of $N \rightarrow \infty$ elements. Let $m_{T}$ be the total number of elements contained in loops

$$
m_{T}=\sum_{m=1}^{\infty} m n_{m} .
$$

Then the distribution of $\left\{n_{1}, n_{2}, \ldots\right\}$ takes the form

$$
P\left(n_{1}, n_{2}, \ldots\right)=\frac{m_{T}}{N} \exp \left[-m_{T}^{2} /(2 N)\right] \prod_{m=1}^{\infty} \frac{\left(1 / m^{n_{m}}\right)}{n_{m} !} .
$$

Thus one can have reasonably long loops with a maximum likely length of order $N^{1 / 2}$. The linkage loops do not determine the cycle structure. To know the number and kind of cycles, one has to know the coupling functions. For a given loop to make a non-trivial cycle, all the functions on it must be either identity or negation, for if there is a constant function in the loop, one element has a fixed value and it passes on its constancy to all the other elements in the loop. So think of a specific case. Let us have a loop, with $m=3$, in which element 1 is the ancestor of element 2 which is the ancestor of element 3 which is the ancestor of element 1 . Let all the coupling functions be identity. Then the initial data just cycles around the loop. If the initial data is $(A B C)$ then next is $(C B A)$, etc. There are two cycles of length one in which all elements are identical and two of length three in which they are not. On the other hand, if all the coupling functions of this three elements are negation, there is one cycle of length two and one of length six. All the different situations are similarly easy to analyze.

Despite the fact that the Kauffman net can contain very large linkage loops for $K=1$, the cycles are reasonably short. Fig. 3.3a shows the distribution of cycle lengths in a Kauffman net composed of $N=15$ elements, and Fig. 3.3b shows the probability $W(n)$ that an arbitrary state belongs to a basin of attraction of size $n$. For $N=15$, there are $2^{15}=32768$ different states. Nonetheless, as can be seen from the figure, the probability of finding a cycle with length bigger than 10 is negligibly small. The reason is that the probability that a loop is relevant (has only non-constant functions) decreases exponentially as the loop length increases. In the usual calculation one assigns the $K=1$ functions with equal weight. If either of the two constant functions are present in the loop, the cycle length is unity. If $a$ is the probability of assignment of the constant functions and $b=1-a$ is the 

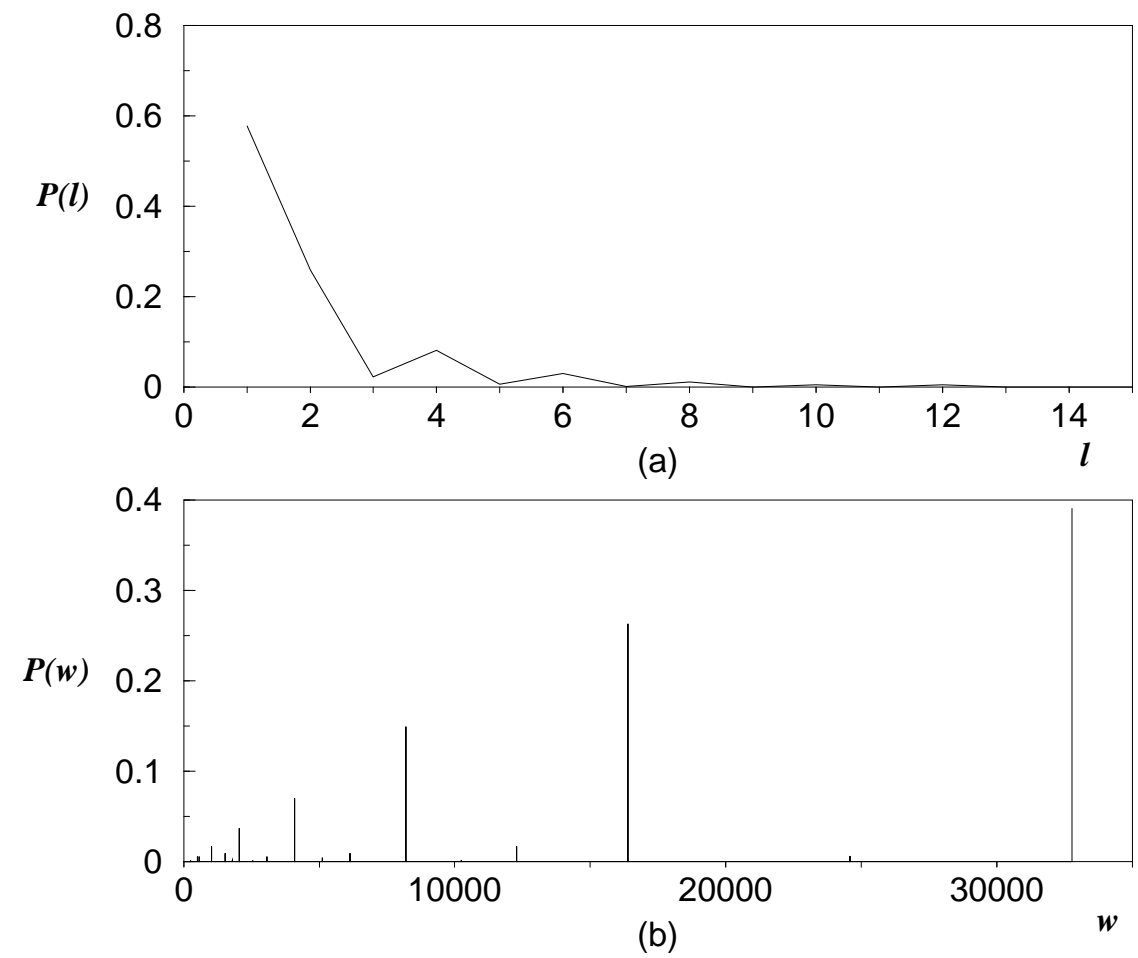

FIGURE 3.3. (a) Distribution of the probability that a cycle has length $l, P(l)$, as a function of $l$ for a Kauffman net of $N=15$ elements and $K=1$. Note that the probability of having cycles with lengths larger than 10 is rather small. (b) Probability $P(w)$ that an arbitrary state belongs to a basin of attraction of size $w$, also for $N=15$ and $K=1$.

probability of assignment of the other two functions, identity and negation, the probability of finding a cycle of length $l$ is proportional to $b^{l}$. In most calculations $b$ is $1 / 2$, so that the probability of really long cycles falls off exponentially in $l$ (this is the justification of equation (3.1)). Flyvbjerg and Kjaer [1988 point out that in the special case with $b=1$, the probability of long cycles falls algebraically. They speculate that the behavior in this limit might be, first, analyzable, and second, very similar to the behavior of the Kauffman net in the critical case $\left(K=K_{c}\right)$ described below. As far as we know, these speculations remain unproven.

\subsection{4 $K \geq N$}

Another case in which it is possible to analyze the structure of the cycles in great detail is the one in which each coupling function depends upon all 
the values of all the different elements in the system. This case has the simplifying characteristic that a change in a single spin changes the input of every coupling function. Therefore, one can analyze some features of the behavior of the system as if the system were annealed rather than quenched. In particular, one can calculate probabilities for hopping from configuration to configuration as if the system were undergoing a random walk through a space of size $\Omega$. The quenched nature of the system only asserts itself when the hopping takes onto a configuration previously visited. After that, one can be sure that the subsequent behavior will be cyclic. There are almost classical mathematical analyses of this situation (Harris [1960); Derrida and Flyvbjerg $[1987 \mathrm{~b}]$ ). We describe this case by considering the calculation of typical distributions of cycle lengths and of transients. Imagine a Kauffman net with $K \geq N$. Imagine that we start from a random configuration $\Sigma_{0}$ at time $t=0$. At subsequent times, we step by step follow the dynamics and essentially go through a random walk $\Sigma_{0}, \Sigma_{1}, \Sigma_{2}, \ldots$, through the configuration space, which has size $\Omega$. This walk continues until we land upon a point previously visited. Let us define $q_{t}$ as the probability that the trajectory remains unclosed after $t$ steps. If the trajectory is still open at time $t$, we have already visited $t+1$ different sites (including the sites $\Sigma_{0}$ and $\left.\Sigma_{t}\right)$. Therefore, there are $t+1$ ways of terminating the walk at the next time step and a relative probability of termination $\rho_{t}=(t+1) / \Omega$. The probability of still having an open trajectory after $t+1$ steps is

$$
q_{t+1}=q_{t}\left(1-\rho_{t}\right)=q_{t}\left(1-\frac{t+1}{\Omega}\right) \text { with } q_{0}=1,
$$

while the probability $p_{t+1}$ of terminating the excursion at time $t+1$ is

$$
p_{t+1}=\frac{t+1}{\Omega} q_{t}
$$

To obtain $P(L)$, the probability that a given starting point is in the basin of attraction of a cycle of length $L$, we note that a closure event at time $t$ yields with equal probability all cycle lengths up to $t$. Therefore,

$$
P(L)=\sum_{t=L}^{\Omega} \frac{p_{t}}{t}
$$

which, in the limit of large $\Omega$ can be approximated by

$$
P(L) \approx \int_{L}^{\infty} \frac{1}{\Omega} e^{-x(x-1) /(2 \Omega)} d x
$$

${ }^{6}$ The results of this section have been known for quite some time; see references Harris [1960]; Derrida and Flyvbjerg [1987b], which study what is called the random map model. Here one studies systems in which one has a random map from point to point in configuration space. The form of argumentation in this section closely follows Coppersmith, Kadanoff, and Zhang [2001a].

Version Jan. 21, 2002...........Edited by : Typeset on 30 October $2018-2 \mathrm{~h} 18$ 
It is also important to consider the probability $P(m, L)$ of finding a cycle of length $L$ after having gone through a precursor of length $m$, given by

$$
P(m, L)=\frac{1}{\Omega} q_{m+L-1} .
$$

(The factor $1 / \Omega$ comes from the fact that only one point, $\Sigma_{m}$, of the state space can split the entire sequence $\left\{\Sigma_{0}, \Sigma_{1}, \ldots, \Sigma_{m}, \Sigma_{m+1}, \ldots, \Sigma_{m+L}\right\}$ of $m+L$ states into two pieces of lengths $m$ and $L$ respectively.) In the limit of large $\Omega$, the previous expression can be approximated by

$$
P(m, L) \approx \frac{\exp \left[-(m+L)^{2} /(2 \Omega)\right]}{\Omega} .
$$

The most important characteristic of the results (3.6) and (3.7) is that the typical cycle length and the typical precursor length are each of order $\Omega^{1 / 2}$. Thus, a very small fraction of the total configurations participate in each transient or cycle, but nonetheless the cycles and the transients may be very long. There is another, and very nice, interpretation of the results just calculated. If the precursor length is zero, equation (3.7) gives the probability that our system will contain a cycle element in a cycle of length $L$. Since there are $\Omega$ possible starting points, the average number of cycles of length $L$ in our system is

$$
\left\langle N_{c}(L)\right\rangle=\frac{\exp \left[-L^{2} /(2 \Omega)\right]}{L} .
$$

Here the $\langle\cdots\rangle$ represents an average over realizations. An integration then gives us the information that the average total number of cycles is proportional to $\ln \Omega$, or more precisely ${ }^{7}$

$$
\left\langle N_{c}\right\rangle=\frac{\ln 2}{2} N+\mathcal{O}(1)
$$

Fig. 3.4 a shows the distribution $P(L)$ for a Kauffman net with $N=$ $K=10$, while Fig. 3.4 b shows the probability $P(w)$ that a given starting point belongs to a a basin of attraction of size $w$. Note that most of the distribution $P(w)$ is concentrated around $w=1$ and $w=\Omega$, being nearly constant (zero) in between. The above reflects the fact that there are large fluctuations in the number of cycles in different realizations. If the number of cycles were a typical extensive quantity, its median would be the same as its average value. Instead, for large $N$ its median value is half the average, indicating that, in the Kauffman net, the average is dominated by a few situations with anomalously many cycles Harris [1960; Derrida

7 Another result, often reported in the literature Zoli, Guidolin, Fuxe, and Agnat [1996], or table 1 in Kauffman [1990) is $\left\langle N_{c}\right\rangle=N / e$. We do not know the justification for this, and suspect that it is wrong.

Version Jan. 21, 2002...........Edited by : Typeset on 30 October $2018-2 \mathrm{~h} 18$ 

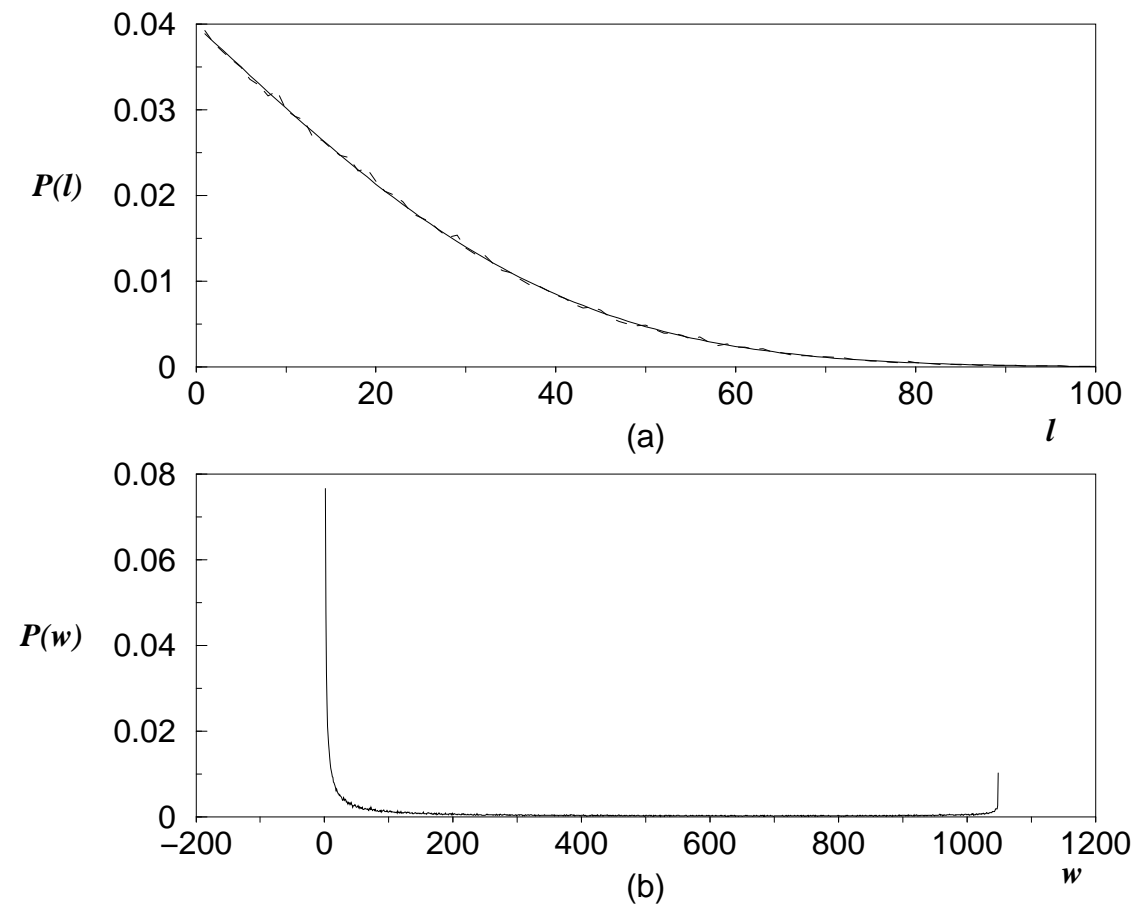

FIGURE 3.4. (a) Probability distribution of cycle lengths for a Kauffman net with $N=K=10$. The solid curve is the theoretical result (3.5) and the broken line is the result of our numerical simulation. (b) Probability that a given starting point $\Sigma_{0}$ belongs to a basin of attraction of size $w$, also for the case $N=K=10$.

and Flyvbjerg [1987b]). The typical size of the largest basin is given by dividing the entire space (of size $\Omega=2^{N}$ ), by the number of cycles (of order $N$, which is actually of order one, given the variance involved with the various fluctuations). To form a basin of size $\Omega=2^{N}$, one starts from the cycle with its typical size $2^{N / 2}$ and count backward finding the set of all first order ancestors, then backwards again to find their ancestors, which are second order ancestors of the cycle, and so on. Since these classes are non-overlapping and bounded in size, after some number of steps (perhaps of order $2^{N / 2}$, the typical precursor size) the classes will shrink in size and eventually one will terminate on one or a few of the most remote ancestors. The total basin size is the sum of the number of elements in these ancestor classes. Since each configuration may have no ancestors, one ancestor or many ancestors, this backward-stepping, ancestor-counting process will have a character similar to a multiplicative random walk. As such it is an inherently highly fluctuating process. So we might expect huge fluctuations in the basin sizes (see Fig. 3.4 b). 


\subsection{Different Phases - Different Cycles}

\subsubsection{Frozen Phases}

In the frozen phase, information typically propagates from a given element to only a very few other elements. Thus a change in initial data will typically affect the subsequent behavior of only a few elements. Similarly, damage to a single coupling function will produce changes which propagate to only a limited number of elements in the system. In these respects the lattice $N-K$ models and the Kauffman net are very similar. However, in other respects

they are very different. For example, in the lattice system, the number of relevant modules is proportional to $N$, so each initially different element has a nonzero chance of being in a relevant module, and each difference at time zero has a nonzero probability of developing into a difference at infinite time. In contrast, in the Kauffman net there are typically only a few short cycles and the overlap rapidly approaches unity, no matter what its initial value might have been. For the $N-K$ model on the lattice there are many loops. In one dimension with nearest neighbor couplings all loops have length unity, but with longer range couplings and in more dimensions some loops might be quite long. Despite the fact that at any given point in the lattice it is exponentially improbable to find long loops, the argument of section 3.3 .1 indicates that the average loop size might well vary algebraically with the size of the system.

1. Lattice system. For small $K$, the lattice system falls into a phase in which there are many short cycles. The number of cycles grows as a power $\mu$ of the volume of the state space, $\Omega$; thus, the typical basin size will be $\Omega^{1-\mu}$. The typical cycle length grows as a power of $N$, with multiplicative fluctuations of order $\ln N$. The growth in cycle length occurs because different modules will have sub-cycles of different lengths. The entire cycle length is the product of the prime factors coming from the modules. Large primes are exponentially unlikely in a given region, but the number of regions observed grows with $N$.

Compare the time development coming from two starting configurations in which some small fraction of elements, spread out through the system, are different. These differences will each have a finite probability of causing a different cyclical behavior, localized in its particular region of the lattice. Independent of the size of the system, at long times the Hamming distance will go to a constant $D^{*}$, proportional to $D(0)$ with a constant of proportionality of order one.

2. Kauffman net. Because the Kauffman net has many fewer relevant modules than the lattice system, even for large $N$, one will be able to observe realizations which always relax into a time-independent behavior, a cycle of length one. According to Bastolla and Parisi [1998b],

Version Jan. 21, 2002...........Edited by : Typeset on 30 October $2018-2 \mathrm{~h} 18$ 
the average number of cycles observed in a given realization will be independent of $N$. Realizations with long cycles will be exponentially unlikely. However, when one gets a long cycle, one can expect to have very many of them. The frozen phase is one in which almost all the variables end up with fixed values. That is, for a given realization, after a large number of steps most variables approach a value which is independent of the initial state of the system. Thus the system behaves, for most of the variables, as if it were highly frictional with the result that each variable comes to a stop its own 'best' value. This is the reason that the overlap goes to one as time goes to infinity in this phase.

The majority of our knowledge of the Kauffman-net behavior of the frozen phase comes from two calculations. As discussed above, the Kauffman net with $K=1$ was solved exactly by Flyvbjerg and Kjaer (Flyvbjerg and Kjaer [1988]). For the case $K=2$, Lynch [1993b] has proven a group of theorems which apply on the frozen side of the $K=2$ system. To ensure the system was frozen, Lynch looked at the case in which there is unequal weight to the four classes of $K=2$ functions, and demanded that the constant functions have a larger weight than the non-canalizing ones. This ensured that the Hamming distance, as calculated by an approach like that in equation (2.4), would decay exponentially. He then proved that the system was in a frozen phase by showing:

1. Almost all elements in the system were in their stable state.

2. Almost all gates were weak, that is, changing their value does not affect the cycle that is entered.

3. The length of the transient, i.e. the number of steps before the system enters its cycle, is bounded below by a constant times $\ln N$.

4. There is also a rather strong bound on the cycle length. The bound includes the statement that the median cycle-length is bounded by a number which is independent of $N$.

\subsubsection{Chaotic Phases}

In contrast to the frozen phase, the chaotic phase is one in which a nonzero fraction of the variables remain sensitive to initial conditions. In fact, most variables keep changing their values throughout the time development of the system. In the chaotic phase the average length of limit cycles and of the transient which occurs before the entry of the limit cycle both grow exponentially with $N$. Because of this explosive growth as the system is made larger, numerical investigations of orbits in the chaotic phase are limited to small system sizes (see, e.g., Bastolla and Parisi [1998b]).

Version Jan. 21, 2002...........Edited by : Typeset on 30 October 2018 - 2h18 
As discussed above, the case when each input is coupled to all the others can be solved exactly (Derrida and Pomeau [1986); Derrida and Flyvbjerg [1987b]), with the typical cycle length growing with system size as $2^{N / 2}$. When $K$ is finite but greater than the critical value $K_{c}$, both the Kauffman net and the lattice models have typical cycle lengths that grow exponentially with $N$. This behavior reflects a complex network of dependency loops in this regime. Though it is plausible that the details of the interconnected loop structures could be different for lattice models and for the Kauffman net, they do not appear to lead to marked differences in the behavior of the two types of models in the chaotic regime.

\subsubsection{Critical Behavior}

We have already seen that there is a close connection between the phase transition of the $N-K$ model and the standard percolation transition of statistical physics. According to Obukhov and Stauffer [1989], random Boolean networks on nearest-neighbor d-dimensional lattices belong to the "universality class' of directed percolation with quenched disorder in d+1 dimensions. However, this result is an argument, not a theorem. The sharing of the universality class means that the $N-K$ models share many of the detailed properties of correlation and ordering with the directed percolation models of standard statistical mechanics. More particularly, the annealed $N-K$ model based upon nearest neighbor interactions is equivalent to a directed percolation problem (Derrida and Stauffer [1986]). Moreover, when the interaction range is infinite (roughly corresponding to infinite dimensions or a simplectic geometry), the quenched (or usual) $N$-K model is equivalent to the corresponding directed percolation problem Derrida and Pomeau [1986]; Derrida and Weisbuch [1986]).

Many studies have been carried out to determine the statistical properties of cycles in the critical phase. The main results can be summarized by saying that in the critical phase, both the typical cycle lengths as well as the mean number of different attractors grow algebraically with $N$. More explicitly, it was believed that these quantities were proportional to $\sqrt{N}$ (see for instance, Bastolla and Parisi [1998b; ; Flyvbjerg [1989; Kauffman [1990, 1969, 1995, 1993]). This was one of the most attractive results of the $N-K$ model in that it matched one of the power-law behaviors exhibited by living organisms, as we will see in section 6.1. However, S. Bilke and F. Sjunnesson have recently brought into question the $\sqrt{N}$ dependence of the mean number of different attractors, arguing that this result comes as a consequence of a biased undersampling of the whole state space Bilke and Sjunnesson [2001]).

Because the size of the state space grows exponentially with the number of elements $N$, it is not feasible to enumerate completely all the different cycles in a given network realization unless $N$ is quite small. What people usually do is to probe the state space with a small fraction of its elements

Version Jan. 21, 2002 ...........Edited by : Typeset on 30 October $2018-2 \mathrm{~h} 18$ 


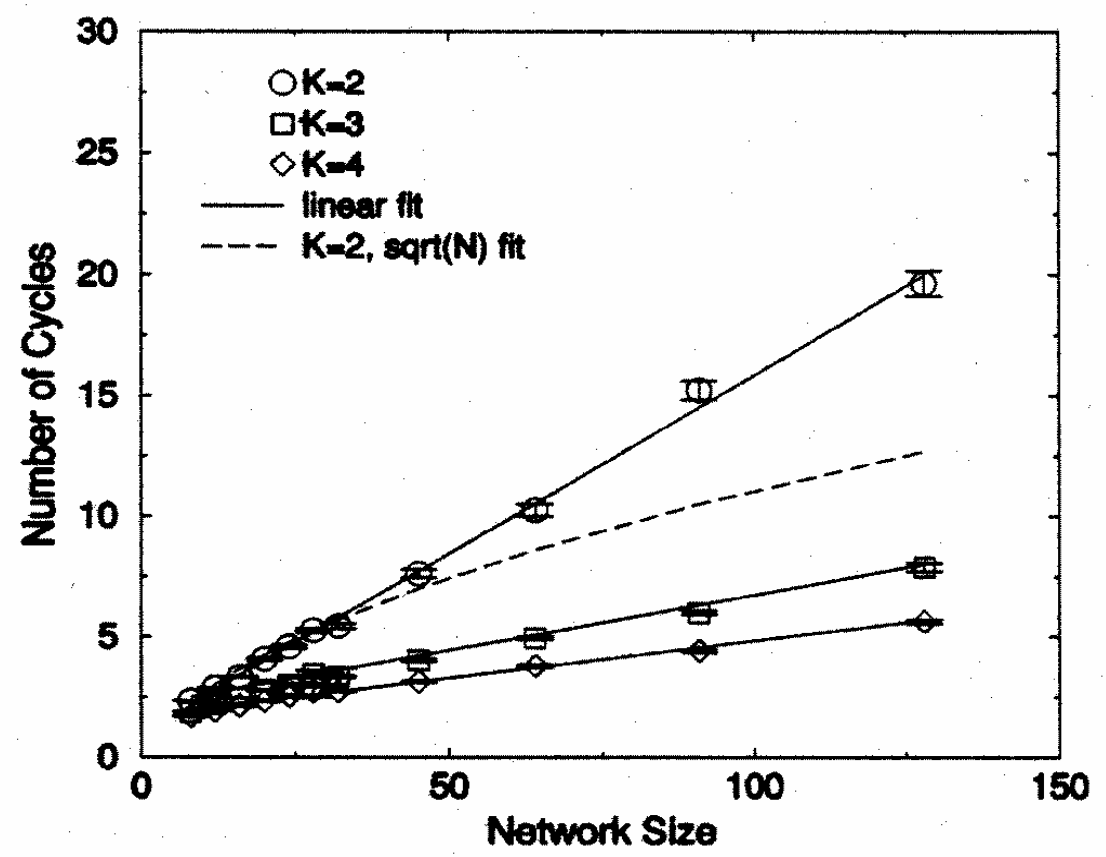

FIGURE 3.5. Mean number of different limit cycles as a function of the network size for critical Kauffman networks with connectivities $K=2,3,4$. The solid curves are the best linear fits to the numerical data, whereas the dashed curve is a $\sqrt{N}$ fit for the case $K=2$. Taken from Bilke and Sjunnesson [2001]

(which are assumed to be representative), and then to infer the statistical properties of the model from this sampling. However, this method, which has yielded the $\sqrt{N}$ dependence referred to above, has the problem that it is possible to miss a small but still important fraction of cycles.

In their work, Bilke and Sjunnesson (ibid.) use a different approach. They present a decimation method which eliminates the stable elements of the network retaining only the relevant ones. Since for any realization of the network the number of different cycles as well as their lengths depend only on the relevant elements, all the statistical information related to limit cycles is preserved through this decimation procedure. The advantage in eliminating the stable elements is that the number of variables is drastically reduced. As a consequence, it is much easier to perform a full enumeration study of the number of different cycles for each realization of the network. Through this approach, Bilke and Sjunnesson found that, in the critical phase of the Kauffman net, the mean number of different attractors $\left\langle N_{c}\right\rangle$

\footnotetext{
${ }^{8} \mathrm{~A}$ stable element is one which evolve to the same fixed value independently of the initial state.
}

Version Jan. 21, 2002 . .Edited by : Typeset on 30 October $2018-2 \mathrm{~h} 18$ 
grows linearly with $N$ instead of as $\sqrt{N}$. Fig. 3.5 (taken from Bilke and Sjunnesson [2001]) shows the mean number of different cycles $\left\langle N_{c}\right\rangle$ as a function of the system size $N$. As can be seen, the linear dependence $\left\langle N_{c}\right\rangle \sim$ $N$ fits the numerical data much better than the $\sqrt{N}$ behavior.

In addition to the exponential growth of the state space as $N$ increases, the number of network realizations grows superexponentially with $K$. The evidence presented by Bilke and Sjunnesson raises the possibility that some of the results obtained so far related to cycle lengths and basins of attraction could also include a systematic bias due to undersampling. More work is needed to address these questions.

\section{Reversible Models}

Kauffman nets are generic models for $N$ elements coupling $K$ different variables. In this section we specialize this generic model to include an interesting symmetry property: time reversal symmetry. The discrete symmetry and quenched randomness together cause some new properties to emerge.

The standard Kauffman net that we have considered so far, whose dynamics is given by equation (1.1), is dissipative because multiple different states can map into one, so that information is lost. In this section we will refer to this system as the dissipative Kauffman net or simply as the dissipative $N-K$ model. Not all systems are dissipative; many of the systems considered in Hamiltonian mechanics are reversible. Reversible systems have the property that some transformation of the coordinates (for example changing the sign of all velocities) makes the system retrace perfectly its former path. This section discusses some aspects of the behavior of discrete reversible maps. In the time-reversible Boolean network studied in Coppersmith, Kadanoff, and Zhang [2001a b], the state of the system at time $t+1$ is governed by the equation

$$
\sigma_{i}(t+1)=f_{i}\left(\sigma_{j_{1}(i)}(t), \ldots, \sigma_{j_{K}(i)}(t)\right) \oplus \sigma_{i}(t-1),
$$

where the $\oplus$ denotes addition modulo 2. Each time-reversible network realization has a corresponding dissipative realization with the same functions and connections. In the dissipative $N-K$ model, the state $\Sigma_{t+1}$ is completely determined by the previous state $\Sigma_{t}$. Nevertheless, from equation (4.1) we see that in the reversible model both $\Sigma_{t-1}$ and $\Sigma_{t}$ are required to calculate $\Sigma_{t+1}$. Thus, in the reversible model the state of the system at time $t$ is represented as

$$
S_{t}=\left(\begin{array}{c}
\Sigma_{t-1} \\
\Sigma_{t}
\end{array}\right),
$$

and the state space has now $2^{2 N}$ points. The behavior of the reversible model is in some ways closely analogous and in other ways quite different

Version Jan. 21, 2002 ..........Edited by : Typeset on 30 October $2018-2 \mathrm{~h} 18$ 
than that of the dissipative model. Both models exhibit a phase transition at $K_{c}$, a critical value of $K$ below which the system "breaks up" into disconnected sections, and above which there is a percolating cluster of connections. The value of $K_{c}$ is slightly lower for the reversible model $\left(K_{c} \approx\right.$ 1.6) than for the corresponding dissipative model $\left(K_{c}=2\right)$, and some details of the transition are different. But for both models the observed behavior is consistent with a percolation picture. For $K<K_{c}$, changing one element of a system leads to changes in only a finite number of other elements, whereas for $K>K_{c}$, changing one element causes a cascade of influence that spreads to a nonzero fraction of all the other elements. The typical cycle length grows slower than linearly with the size of the system when $K<K_{c}$, and exponentially with system size when $K>K_{c}$.

One big difference between the reversible and dissipative models is that they have vastly different numbers of attractors (or, in the reversible case, limit cycles). Large differences in the behavior are not entirely unexpected because in the dissipative model many different state space points end up at the same attractor, whereas in the reversible model every state space point is on exactly one limit cycle. For example, when $K=0$ (all input functions either 1 or 0 ), the usual Kauffman net has only one attractor, while the number of limit cycles of a reversible Kauffman net is proportional to $2^{2 N}$. The reversible result can be understood by noting that for either input function, each element is in one of four different cycles, depending on its initial conditions, and that when $K=0$ the elements are all independent. In the other limiting case $K=N$, as discussed above, the number of attractors of a dissipative Kauffman net is proportional to $N$ (see equation (3.9)). In contrast, in the reversible model with $K=N$, and indeed throughout the regime $K>K_{c}$, the number of limit cycles grows as $2^{N}$.

The number of attractors in the reversible model with $K=N$ can be understood by studying the mechanisms that lead to orbit closure. One way to close an orbit is to repeat two successive configurations, so that $\Sigma_{T}=\Sigma_{0}$ and $\Sigma_{T+1}=\Sigma_{1}$. Using the approximation that each successive $\Sigma$ is chosen randomly from the $2^{N}$ possibilities yields a probability of an orbit closure at a given time of order $\left(2^{-N}\right)^{2}=2^{-2 N}$. However, there is another mechanism for orbit closure that leads to much shorter orbits and thus totally dominates the behavior. If at some time $\tau$ one has $\Sigma_{\tau}=\Sigma_{\tau-1}$, then time-reversibility implies that $\Sigma_{\tau+n}=\Sigma_{\tau-1-n}$ for all $n$. Similarly, if $\Sigma_{\tau+1}=\Sigma_{\tau-1}$, then $\Sigma_{\tau+n}=\Sigma_{\tau-n}$ for all $n$. Because the orbits reverse at these points, we call them "mirrors." Once two mirrors have occurred, then the orbit must close in a time that is less than the time it has taken to hit the second mirror. Again assuming that each successive $\Sigma$ is chosen randomly from the $2^{N}$ possibilities, one finds that a mirror occurs at a given time with probability proportional to $2^{-N}$, so the expected number of steps needed to hit two mirrors is of order $2^{N}$. Hence, typical orbit lengths are of order $2^{N}$. Since there are $2^{2 N}$ points in the state space altogether, the number of limit cycles is proportional to $2^{N}$. In the reversible Kauffman 
net, when $K$ is finite but greater than the critical value $K_{c}$, the distribution of cycle lengths can be extremely broad. For example, when $N=18$ and $K=2$, the median cycle length $\bar{l}$ is approximately $\bar{l} \approx 140$, and yet the probability of observing an orbit of length $10^{10}$ is greater than $10^{-4}$. This huge variability arises because of a nontrivial interplay between the discrete symmetry and the quenched randomness in the system. The occasional extremely long orbit arises because some combinations of coupling realizations and initial conditions are such that mirrors cannot occur at all. If the mirrors are not available to close the orbits, then the system must wait until two successive configurations happen to repeat. In Coppersmith. Kadanoff, and Zhang [2001a ) it is shown that in a system with finite $K$ in the true thermodynamic limit, almost all realizations and initial conditions yield no mirrors, and typical orbit lengths grow as $2^{2 N(1-\epsilon)}$, where $\epsilon$ is of order $1 /\left(2^{2^{K}}\right)$. However, the crossover to the limiting behavior occurs only when $N \sim 2^{2^{K}}$, so that even for moderate $K$, this behavior is not accessible numerically (for example, when $K=3$, one requires $N \sim 256$, a value at which $2^{N} \sim 10^{77}$ and $\left.2^{2 N} \sim 10^{154}\right)$.

The significance of this enormous variability in the cycle lengths is not clear. One way to interpret these results is to conclude that characterizing cycles is not the right way to study the model. It would be interesting to investigate whether enormous variability arises in other random systems with discrete symmetries, and to determine whether there are possible experimental consequences. One possible starting point for comparison is with properties of random magnets, for which regions of atypical couplings lead to Griffiths singularities (Griffiths [1969), which have stronger effects on dynamic than static properties (Randeria, Sethna, and Palmer [1985)). One must explore whether the analogy is appropriate (this is not obvious because Kauffman networks are not lattice-based), and if so, whether the results for the reversible Kauffman model indicate that dynamics far from equilibrium can be even more strongly affected by atypical coupling realizations than are the properties in spin models that have been studied to date.

Version Jan. 21, 2002...........Edited by : Typeset on 30 October $2018-2 \mathrm{~h} 18$ 


\section{Beyond Cycles}

\subsection{Non-robustness of Cycles}

A cycle forms when the system returns to a configuration which is exactly the same as one it has previously visited. A demand for an exact return should be viewed not as a single constraint but in fact as $N$ constraints upon the configuration. Such a strict demand makes the properties of cycles quite special and delicate. For this reason, a study of cycles is probably not what one would want for understanding the possible physical or biological consequences of the models like the $N$-K model. The statement just made flies in the face of a very large body of work, some of which we have just described. We should, for this reason, argue for this statement with some care.

Why don't we believe in cycles?

1. The characterizers of cycles are neither intensive nor extensive variables.

2. With exponentially short cycles in localized regions one gets power laws overall.

3. The critical situation has very many, short cycles which are not observed when one starts from randomly picked starting points.

4. Attractor basins are complex in character, being at best multi-fractal for large chaotic systems.

5. In a large system you must wait so long to see a cycle that it cannot be really important.

6. In a large chaotic system, changing one rule changes the cycles quite a bit.

7. In both glasses and biological systems one wants to characterize the system by things which are very robust.

We do believe that generic properties of cycles are important though, in that they characterize general aspects of dynamical systems. Nonetheless, the huge fluctuations throughout realizations in such important quantities as cycle lengths and number of different attractors, calls for other types of characterizations. Real networks, whether they are genetic or neural or of any other kind, are always subjected to external perturbations. The robustness in the dynamics of the network can not rely on quantities which change dramatically with perturbations. Hence, it is important to characterize the dynamical properties of the network in the presence of noise, trying to find out which kind of quantities are preserved under the influence of noise, and which ones are not.

Version Jan. 21, 2002...........Edited by : Typeset on 30 October $2018-2 \mathrm{~h} 18$ 


\subsection{Characterization via noise}

The addition of noise to a map provides a possibility for generating additional information about the behavior of the models. Noise naturally blurs out the sharpness of behavior, making for a more "fuzzy" characterization. Noise is, then, a natural way to get away from the difficulty posed by the overly-precise characterization provided by the cycles.

Unfortunately, most previous work on the $N-K$ model does not include noise. We do wish to point to two papers (Miranda and Parga [1989; Golinelli and Derrida [1989) in which noise has been used to probe $N-K$ behavior. For reasons which will become more evident later on, we describe these papers respectively as a "crossing paper" and a "convergence paper". These papers both break the precision of the dynamical rule of equation (1.1) by saying that the rule is broken with a probability $r$

$$
\sigma_{i}(t+1)=\left\{\begin{array}{llc}
f_{i}\left(\sigma_{j_{1}(i)}(t), \ldots, \sigma_{j_{K_{i}}(i)}(t)\right) & \text { with probability } & 1-r \\
\neg f_{i}\left(\sigma_{j_{1}(i)}(t), \ldots, \sigma_{j_{K_{i}}(i)}(t)\right) & \text { with probability } & r
\end{array}\right.
$$

which can also be written in the alternative form:

$$
\sigma_{i}(t+1)=\left\{\begin{array}{llc}
f_{i}\left(\sigma_{j_{1}(i)}(t), \ldots, \sigma_{j_{K_{i}}(i)}(t)\right) & \text { with probability } & 1-2 r \\
1 & \text { with probability } & r \\
0 & \text { with probability } & r .
\end{array}\right.
$$

This equation can be described as providing probabilities $r$ for the two possible values of the outcome, independently of the value of $f_{i}$. In Golinelli and Derrida [1989] the rules are described in terms of a temperature $T$, related to $r$ by

$$
r=\frac{1-\tanh (1 / T)}{2}
$$

Both groups examine the development of two or more different initial configurations using the same realizations. They also apply exactly the same rules and the same probabilistic choices to the different configurations. So far, both papers are essentially similar. There are two kinds of differences, the first being the choice of measurement, and the second being the way they apply equations (5.1) and (5.2).

The convergence paper starts with two or more randomly chosen initial configurations, $\Sigma_{0}^{1}, \Sigma_{0}^{2}, \ldots, \Sigma_{0}^{m}$, and calculates the resulting trajectories step by step:

$$
\begin{aligned}
\Sigma_{0}^{1} & \rightarrow \quad \Sigma_{1}^{1} \rightarrow \Sigma_{2}^{1} \rightarrow \ldots \Sigma_{\tau}^{1} \\
\Sigma_{0}^{2} & \rightarrow \quad \Sigma_{1}^{2} \rightarrow \Sigma_{2}^{2} \rightarrow \ldots \Sigma_{\tau}^{2} \\
& \ldots \\
\Sigma_{0}^{m} & \rightarrow \Sigma_{1}^{m} \rightarrow \Sigma_{2}^{m} \rightarrow \ldots \Sigma_{\tau}^{m}
\end{aligned}
$$

Version Jan. 21, 2002............Edited by : Typeset on 30 October $2018-2 \mathrm{~h} 18$ 
At each step, and for each $i$, a choice is made among the three branches of equation (5.2), and that choice is equally applied to the $m$ configurations. 9 The calculation continues until two of the $m$ configurations become identical (say for example $\Sigma_{\tau}^{1}=\Sigma_{\tau}^{2}$ ). The time needed to achieve the convergence is noted. We will denote this time by $\tau_{m}$, stressing the fact that $m$ configurations are being analyzed.

In some ways, the convergence calculation is more complicated than the crossing one. The noise as defined by equation (5.2) tends to produce convergence because it is applied equally to all trajectories and because makes the values of the elements to be equal in all trajectories. In the limit of infinite $N$, the system shows three phases: the low noise phase in which almost always trajectories will not converge, the high noise phase in which trajectories will always converge, and a separating critical phase. These are respectively described as low temperature, high temperature, and critical phases.

The authors examine $K=4$ and large- $N$, picking the noise-levels $r=$ 0.15 (low temperature phase), $r=0.25$ (critical phase), and $r=0.35$ (high temperature phase). Without noise, the $N-K$ model would show chaotic behavior, and very long cycles. In the presence of noise the average of $\ln \tau$, with $\tau$ being the convergence time, diverges as $N$ goes to infinity. The behavior of the convergence time for two orbits, $\tau_{2}$, can be calculated in the annealed sequential update case, and the results compared with simulations for both annealed and quenched system. In both simulation and theory, the three phases are characterized by having different forms of divergence of $\left\langle\ln \tau_{2}\right\rangle$ with $N$ (see Fig. 5.1). At low temperatures the divergence is linear: $\left\langle\ln \tau_{2}\right\rangle \sim N$. In the critical phase $\left\langle\ln \tau_{2}\right\rangle$ diverges linearly with $\ln N$ as $\left\langle\ln \tau_{2}\right\rangle=0.5 \ln N$. The weakest divergence occurs in the high temperature phase in which $\left\langle\ln \tau_{2}\right\rangle$ varies as $\ln \ln N$. These $N$-dependencies describe the variation of the number of elements forming the barrier to having two configurations merge into one another. The noise causes these elements to be identical and then the merge occurs. Thus, for example, in the low temperature phase, we must bring to equality a finite fraction of all the elements in the system in order to have the convergence.

In some loose sense, these numbers measure the size of the barriers which hold together the attractors for this system. But it is hard to know what the attractors themselves might be. Because $r$ is relatively large, these presumed attractors are probably not the cycles of the original system. In fact, the behavior of $\tau_{2}$ is much the same for the annealed system (which has no cycles) as for the quenched system, which does have cycles at $r=0$. Nobody has yet investigated the limiting case as $r$ goes to zero. It might be most interesting to look at this limit, particularly in association with a

\footnotetext{
${ }^{9}$ The two-branch versus three-branch methods become inequivalent when they are applied to several configurations at once.
}

Version Jan. 21, 2002...........Edited by : Typeset on 30 October $2018-2 \mathrm{~h} 18$ 


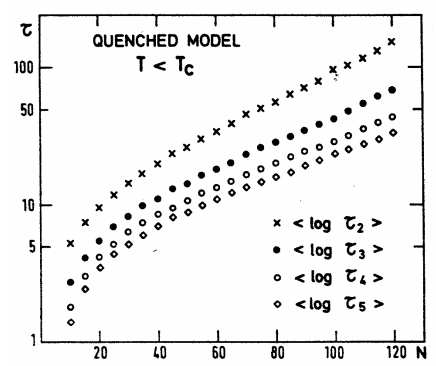

(a)

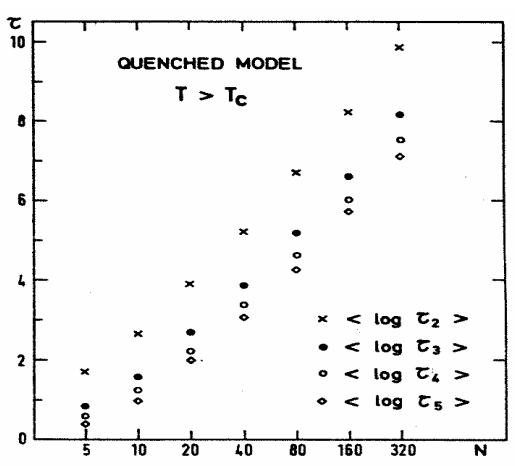

(c)

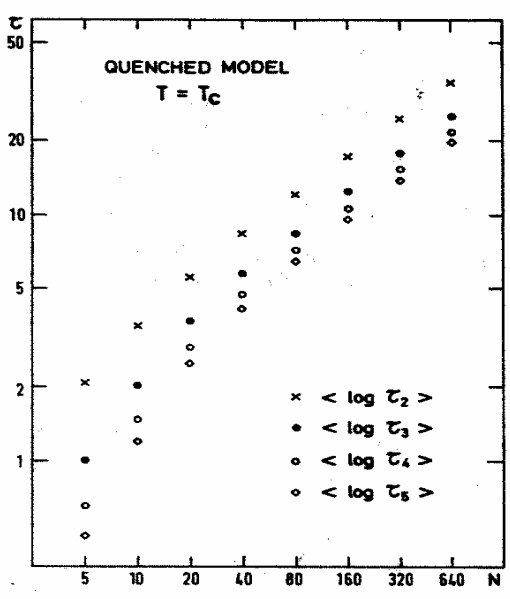

(b)

FIGURE 5.1. Plots of $\left\langle\ln \tau_{m}\right\rangle$ versus $N$, taken from reference Golinelli and Derrida [1989]. In all the cases the connectivity of the network was $K=4$. The three graphs correspond to three values of $r$ : (a) Low temperature phase, $r=0.15$; (b) Critical phase, $r=0.25$; (c) High temperature phase, $r=0.35$.

limit which keeps the system critical (say $K$ goes to 2 ).

Let us consider the case in which more than two trajectories are analyzed. Start with $m$ trajectories and let $\tau_{m}$ measure the first time when any two of these have converged. If there are only $M$ large or important basins, one might well expect $\left\langle\ln \tau_{m}\right\rangle \ll\left\langle\ln \tau_{M}\right\rangle$ for $m>M$. Instead one observes that

$$
\left\langle\ln \tau_{m}\right\rangle=\left\langle\ln \tau_{2}\right\rangle-\ln \frac{m(m-1)}{2},
$$

in both theory (in the annealed approximation) and simulation. This form indicates an indefinitely large number of attractors, all with basins of comparable size.

The crossing paper considers two different configurations $\Sigma_{0}^{1}$ and $\Sigma_{0}^{2}$, which can belong either to different attraction basins or to the same basin of attraction, and then iterates forward, noting all configurations $\left\{\Sigma_{0}^{1}, \Sigma_{1}^{1}\right.$,

Version Jan. 21, 2002...........Edited by : Typeset on 30 October $2018-2 \mathrm{~h} 18$ 


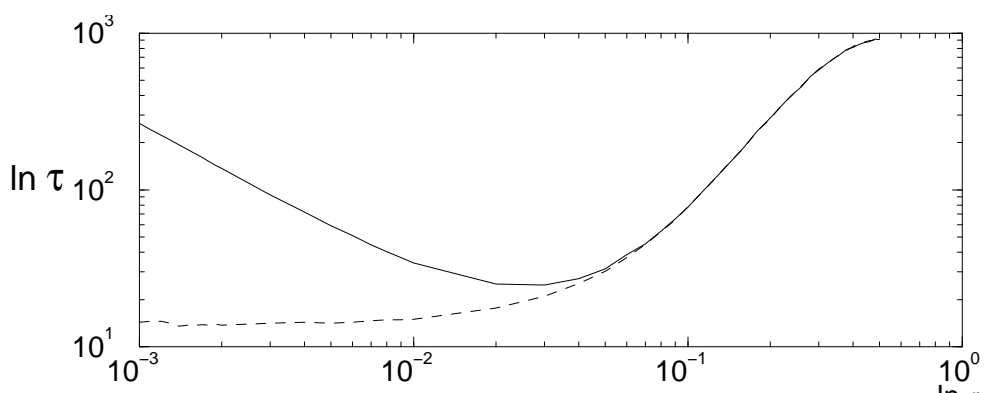

(a)

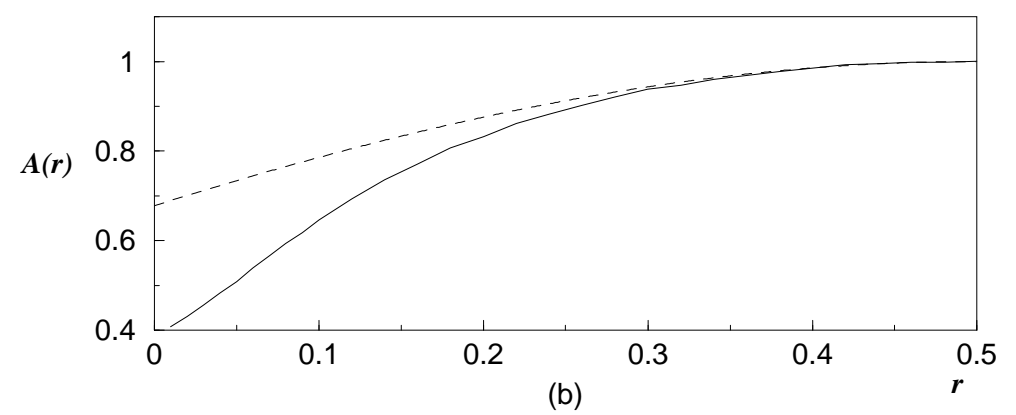

FIGURE 5.2. (a) Log-log plot of the crossing time $\tau$ as a function of the level of noise $r$. The solid curve is $\tau_{d}$, the crossing time starting from different attractors, whereas the dashed curve corresponds to $\tau_{s}$, the crossing time starting from the same attractor. (b) The index $A(r)$ describing the fractal dimension of the attractor plotted as a function of $r$. The solid curve is the result of the numerical simulation, obtained as $A(r)=2 \ln \tau / \ln \Omega$, and the dashed line is the theoretical prediction given in (5.7). Note that these curves become identical when $r \rightarrow 0.5$. Both graphs (a) and (b), calculated for a situation with $K=2$ and $N=20$, were taken from reference Qu, Kadanoff, and Aldana [2002].

$\left.\Sigma_{2}^{1}, \ldots\right\}$ and $\left\{\Sigma_{0}^{2}, \Sigma_{1}^{2}, \Sigma_{2}^{2}, \ldots\right\}$ they produce. In each step of iteration, and for each $i$-value, a choice is made between the two branches of equation (5.1), and that choice is applied to both configurations. This continues until the time $\tau$ in which one of the two trajectories attains a configuration previously entered by the other one (for example, if $\Sigma_{\tau}^{2}$ is equal to one of the configurations $\left.\left\{\Sigma_{0}^{1}, \Sigma_{1}^{1}, \ldots, \Sigma_{\tau}^{1}\right\}\right)$. The calculation is terminated at this crossing event. The measured quantity is the "time" $\tau$ needed to produce the crossing.

In the absence of noise, if two initial configurations belong to different basins of attraction, the time for the two subsequent trajectories to cross is infinite. In the presence of noise, there is a chance for each trajectory to "jump out" of its basin of attraction, exploring a bigger part of the state space. The two trajectories will have a number of opportunities equal to $\tau^{2}$, to cross one another before $\tau$ steps have elapsed. If the size of the space 
being explored by the trajectories is $\Omega(r)$, then the typical time for the crossing will be

$$
\tau \approx \Omega(r)^{1 / 2} .
$$

Miranda and Parga simulated the system and measured $\tau$ as a function of $r$ and $N$ for the critical Kauffman net in which $K=2$. Their result for large $N$ may be summarized as

$$
\Omega(r)=\Omega^{A(r)},
$$

where $\Omega=2^{N}$ is the volume of the state space in the system.

In the work by Miranda and Parga the "fractal" exponent $A(r)$ was not estimated accurately. This work was recently extended by X. Qu et al., who consider larger values of noise and different connectivities of the network (Qu, Kadanoff, and Aldana [2002]). The authors analyze two cases to compute the crossing time, when the two initial configurations belong to the same basin of attraction, and when they belong to different basins. We will denote these two crossing times by $\tau_{s}$ and $\tau_{d}$ respectively. Fig. 5.2a shows the average crossing times $\tau_{d}$ and $\tau_{s}$ as funcions of $r$, for a net with $N=20$ and $K=2$. As can be seen, when $r$ is close to its maximum value 0.5 , both times are practically the same. In fact, $\mathrm{Qu}$ et al. have shown that for large values of $r$ both $\tau_{s}$ and $\tau_{d}$ behave as

$$
\tau_{d, s} \approx \frac{\sqrt{\pi}}{2} 2^{N / 2}\left\{1+\frac{(1-2 r)^{2}}{2^{K}}\right\}^{-N / 2} .
$$

The above expression agrees with equations (5.4) and (5.5) by identifying

$$
A(r)=1-\frac{\ln \left[1+(1-2 r)^{2} / 2^{K}\right]}{\ln 2} .
$$

In contrast, when $r$ is close to 0 the behavior of $\tau_{d}$ and $\tau_{s}$ differ substantially. For $r \rightarrow 0$ the divergence of $\tau_{d}$ is given simply by

$$
\tau_{d} \approx C_{1} / r+\tau_{0}
$$

where $C_{1}$ only depends on $K$ and $N$, and $\tau_{0}$ is the value of $\tau_{s}$ at $r=0$.

The complete analytical expresion of $A(r)$, valid in the whole interval $[0,1 / 2]$ is not known yet. Fig. 5.2 b shows a plot of $A(r)$ obtained by numerical simulations. It is interesting to note that the attractor has a fractal volume which depends upon $r$. Once again, one is frustrated because one does not know what the attractor might be. It is once again probably not anything directly related to a cycle, since starting points in the same cycle or in different cycles both give the same $\tau$-values for the higher values of $N$ and $r$. Here too one might guess that studies with smaller values of $r$ might shed light on the $N-K$ model attractors.

Version Jan. 21, 2002...........Edited by : Typeset on 30 October $2018-2 \mathrm{~h} 18$ 


\section{Applications}

\subsection{Genetic networks and cell differentiation}

$N-K$ models have been widely used in the modeling of gene regulation and control (Somogyi and Sniegoski [1996]; De Sales, Martins, and Stariolo [1997]; Kauffman [1993]; Huang and Ingber [2000]). A very remarkable characteristic of multicellular organisms is that all the different cells of which they are made have the same genetic information. What makes the difference between the different cell types are the genes which are expressed in every cell at every moment. In a given cell type, some particular genes are turned off and others are turned on. So, in a liver cell, only the "liver genes" are being expressed while all the other genes are turned off, whereas in a neuron the "neuron genes" are the only ones which are expressed.

The physical and chemical mechanisms by which the cell determines which genes are to be expressed and which are not are not yet fully understood. There is evidence that gene regulation and control can occur at every stage during the metabolic pathways leading up from the genetic information contained in the DNA, to the translation of this information into proteins. Nevertheless, most of the gene regulation and control seems to occur at the level of transcription of the genetic information. At this level, one gene of DNA is transcribed into a molecule of messenger ARN (mARN) only if the conditions for this transcription are present. In the most simple model (applicable to bacteria), for the transcription of one gene into a molecule of mARN, it is necessary a protein, called activator, which attaches to the beginning of the gene indicating that this gene is ready to be transcribed (see Fig. 6.1 a). On the other hand, there also exist repressor proteins which, when attached to the beginning of the gene, inhibit its transcription, turning the gene off (see Fig. 6.1 b).

In eucaryotic cells the situation is more complicated in that many activator or repressor proteins might be needed to activate or to inhibit the expression of a single gene. For example, it is known that the human $\beta$ globine gene (expressed in red blood cells) is regulated by more than 20 different proteins. Some of these proteins may function as both repressors or activators, depending on how they are assembled.

The activator or repressor proteins of a given gene are themselves specified by some other genes, whose expression, is in turn controlled by other proteins codified by other genes and so on. Genes interact each other through the proteins they specify: the product protein of one activated gene can influence the activation or deactivation of other genes. Similarly, the absence of the product protein of a deactivated gene can influence the activation or deactivation of several other genes. In some particular cases, it is known which gene controls which other one, but in most of the cases the interactions between genes in a given cell type are completely unknown. A picture emerges in which genes are mingled together forming a network

Version Jan. 21, 2002...........Edited by : Typeset on 30 October $2018-2 \mathrm{~h} 18$ 


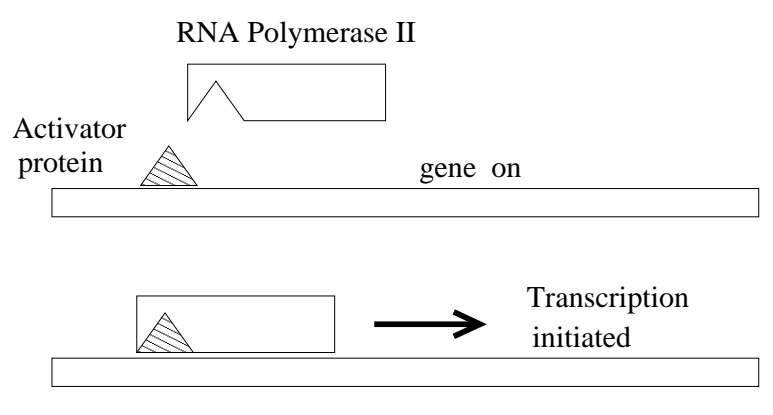

(a)

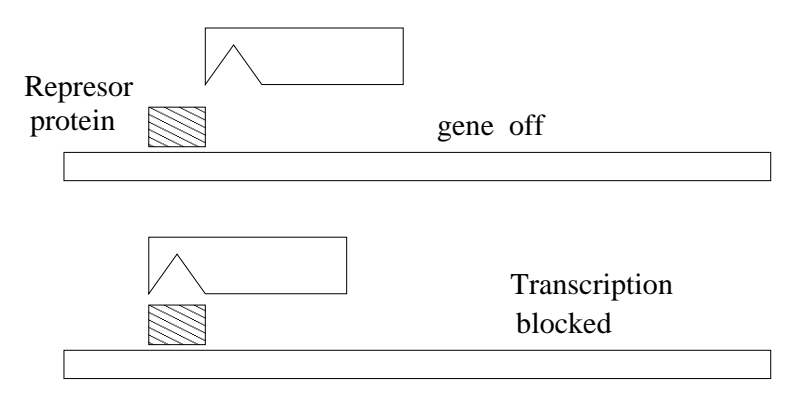

(b)

FIGURE 6.1. Schematic representation of gene regulatory proteins. (a) An activator protein attaches to the gene at a specific binding site, activating the polymerase II which then can transcript the gene into a molecule of mRNA. (b) When a repressor protein is attached to the gene, the polymerase II is blocked up and therefore unable to transcript the information contained in the gene.

of interacting elements which are connected in a very complicated way.

The $N-K$ model was first suggested by Kauffman as a way of modeling the dynamics and evolution of this complicated network of interacting genes (Kauffman [1969). Within this model, two genes are linked if the product protein of one gene influences the expression of the other one. In real cells, genes are not randomly linked. Nevertheless, the web of linkages and connectivities among the genes in a living organism is so complicated (and mostly unknown), that to model these genes as randomly linked seems to be a reasonable approximation. By this means, the results coming out of the model are not restricted to a particular set of linkages and connectivities, but reflect instead the generic properties of this class of networks.

The state of the cell at every moment is determined then by the state of its genes $\sigma_{1}, \ldots, \sigma_{N}$. Before proceeding further, it is important to recall the main assumptions that are usually made to describe the dynamics of

Version Jan. 21, 2002...........Edited by : Typeset on 30 October $2018-2 \mathrm{~h} 18$ 
genetic networks by means of the $N-K$ model:

- Every gene $\sigma_{i}$ can be only in one of two states, turned on (1) or turned off $(0)$.

- Every gene is randomly linked with exactly $K$ other genes.

- The evolution rule $f_{i}$ associated to gene $\sigma_{i}$ is a weighted function which acquires the value 1 with probability $p$ ant the value 0 with probability $1-p$.

- The updating of the network is synchronous.

As we have seen, under these assumptions the phase space breaks up into different cycles or attractors whose properties in the frozen phase and the chaotic phase are substantially different. According to Kauffman's interpretation, each attractor represents a cell type or a cell fate, whereas a single state of the system represents just a temporal state which the cell is passing through. From this point of view, cell reproduction processes start with an initial configuration of genes, which eventually evolves towards its corresponding attractor; the attractor determines a particular cell type or cell fate.

For this mechanism of cell differentiation to be meaningful, the length of the attractors must not be too long, for otherwise the cell would never reach its stable cycle. In the chaotic phase the length of the cycles grows exponentially with the system's size $\left(l \sim 2^{\alpha N}\right.$, where $\alpha$ is of order 1$)$. Therefore, the system has to go through very many states before reaching a stable set of configurations. In addition, systems in the chaotic phase are very sensitive to perturbations (mutations), partially because the number of relevant elements is comparable to the size of the system. The above prompts the thought that genetic networks of living organisms are not in the chaotic phase.

On the other hand, in the frozen phase the cycles are much shorter than in the chaotic phase: an initial set of genes swiftly reaches its stable configurations. The fraction of relevant elements in the frozen phase is close to zero. As a consequence, the system is extremely resistant to point mutations 10 or to damage in one or more of the evolution rules. But in order to evolve, genetic networks of living organisms should allow some degree of sensitivity to mutations, which rules out the frozen phase as a physical state which living organisms could be in.

Kauffman suggested that gene networks of living organisms operate at the edge of chaos (Kauffman [1993), meaning that the parameters have been adjusted through evolution so that these networks are at or near the critical phase. In the critical phase, both the number of different attractors

${ }^{10} \mathrm{~A}$ point mutation is a change in the value of one gene.

Version Jan. 21, 2002 ...........Edited by : Typeset on 30 October $2018-2 \mathrm{~h} 18$ 
as well as their lengths are proportional to a power of $N$, so the cell can reach very quickly its stable configurations. Also, the fraction of relevant nodes in critical networks, even though is small, is not zero, which means that these kinds of networks present some degree of sensitivity to changes in the initial conditions. In other words, critical networks exhibit homeostatic stability, a term which we will come to in the next section.

A very remarkable observation supporting the idea of life at the edge of chaos consists in the fact that the number of different cell types in an organism is roughly proportional to the square root of its DNA content. Furthermore, the mitotic cycle period, which can be considered as a measure of the time required for a cell to reproduce, seems also to be proportional to the square root of the cell's DNA content (Kauffman [1993). Thus, random networks in the critical phase seem to satisfy the requirements of order, evolvability and stability found in living organisms.

Even though this idea is very attractive, there are some problems yet to be solved. As we have mentioned, for unbiased evolution rules $f_{i}$, the critical phase is characterized by the low connectivity $K=2$. This implies that genetic networks of real organisms are restricted to have very low connectivities in order to be at "the edge of chaos". As soon as the connectivity grows, the system becomes more and more chaotic. But it is well known that the connectivity in real genetic networks is rather high. For example, the expression of the even-skipped gene in Drosophila is controlled by more than 20 regulatory proteins, also the Human $\beta$-globine gene we have referred to before (Alberts, Bray, Lewis, Raff, Roberts, and Watson [1994]). In eucaryotes it is common to find that one single gene is regulated by a bunch of proteins acting in association. On the other hand, sometimes when a single signaling receptor protein is activated, it can influence directly the activation of a very large array of genes. Let us consider for instance the activation of the platelet-derived growth factor $\beta$ receptor (PDGFR $\beta$ ), which induces the expression of over 60 genes (Fambrough, Mcclure, Kazlauskas, and Lander [1999]). These examples, among many others, suggest that the connectivity in real genetic networks is not low, but on the contrary, it is very high. Nonetheless, cells do not seem to operate in the chaotic phase.

There are two ways to increase the connectivity in the $N$ - $K$ model without going out of the critical or ordered phase: (a) by the use of weighted evolution functions $f_{i}$, or (b) by the use of canalizing functions. When evolution functions $f_{i}$ are weighted with the probability parameter $p$, the critical line is given by equation (2.8). Fig. 2.4 shows the graph of $K_{c}$ as a function of $p$. Even if we suppose that genetic networks can be either in the frozen phase or in the critical phase, they would be restricted to remain within the shaded area of the figure.

On the other hand, it is known that the fraction of canalizing functions becomes very small as $K$ increases (Kauffman [1984]). An upper bound for 
this fraction is

$$
\frac{4 K}{2^{2^{K-1}}}
$$

which tends to zero as $K \rightarrow \infty$. Consequently, canalizing functions are extremely rare when $K$ is large 11 . If the apparent order seen in living cells relies on either weighted or canalizing functions, it has still to be solved what kind of mechanisms drove, through evolution, the genetic networks towards the generalized use of such type of functions.

Another problem lies in the assumption that genes can be in only two states. It is true that a given gene is expressed or is not. But the product protein of a gene can participate in a variety of metabolic functions, producing even opposite effects depending on the physical and chemical context in which it acts. Such behavior can be modeled by assuming that every gene can acquire more than two states. But in such a case the connectivity of the network must be even smaller to keep the system within the ordered phase. For if we assume that every gene can be, on average, in one of $m$ possible states, and if every one of these states is activated with the same probability, the critical connectivity $K_{c}$ is then given by

$$
K_{c}=\frac{m}{m-1} .
$$

The critical connectivity decreases monotonically when $m>2$, approaching 1 as $m \rightarrow \infty$. The moral is that for this kind of multi-state networks to be in the ordered phase, the connectivity has to be very small, contrary to what is observed in real genetic networks.

Partially to overcome these difficulties, De Sales, Martins, and Stariolo [1997] have proposed a model which is slightly different from the original $N-K$ model. In their model, the dynamics of the system is governed by the equation

$$
\sigma_{i}(t+1)=\operatorname{Sign}\left\{J_{i i} \sigma_{i}(t)+\sum_{l=1}^{K-1} J_{i j_{l}(i)} \sigma_{j_{l}(i)}(t)\right\},
$$

where $J_{i j_{l}(i)}$ is the coupling constant representing the regulatory action of the $j_{l}(i)$ input of gene $i,(l=1,2, \ldots, K-1)$. There is also an autogenic regulation, given by $J_{i i}$. The set of coupling constants $J_{i j}$ represents the very complicated set of biochemical interactions between genes. Since these interactions are mostly unknown, the authors assign the coupling constants in a random way, according to the following criteria:

- The product protein of a given gene can activate, inhibit, or not affect at all the transcription of another gene.

\footnotetext{
${ }^{11}$ Even for $K$ as small as $K=10$, equation (6.1) gives a fraction of canalizing functions of the order $10^{-153}$.
}

Version Jan. 21, 2002...........Edited by : Typeset on 30 October $2018-2 \mathrm{~h} 18$ 


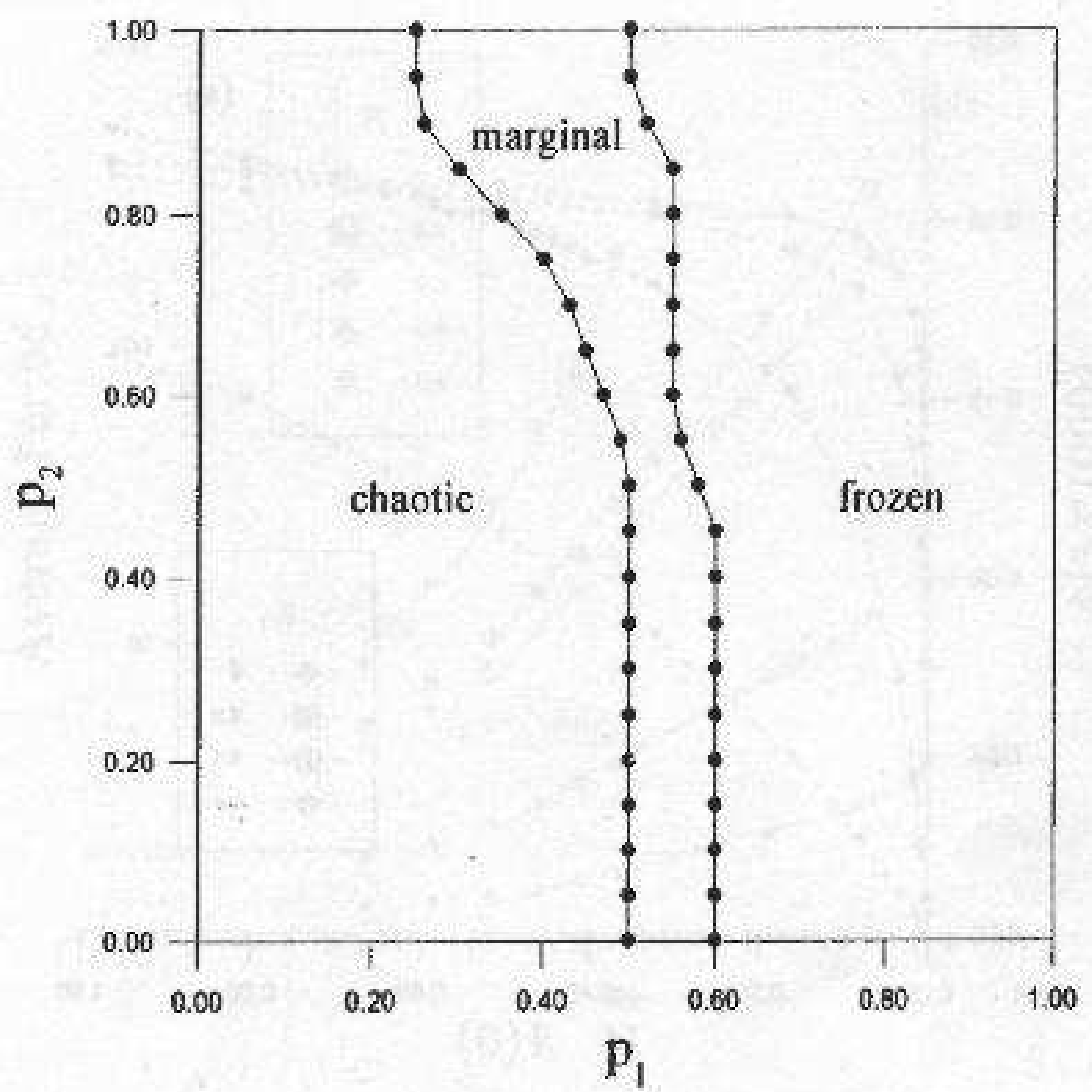

FIGURE 6.2. Phase space for the $N-K$ model with the dynamics given by equation (6.4). As can be seen, under this dynamics the system exhibits three different "phases": frozen, marginal and chaotic (taken from De Sales, Martins, and Stariolo [1997]).

- Interactions between genes are not necessarily symmetrical, namely, $J_{i j} \neq J_{j i}$ in general.

- Autogenic or self-regulation is allowed.

The coupling constants are then assigned according to the probability function given by:

$$
P\left(J_{i j}\right)=\frac{1-p_{1}}{2}\left[\delta\left(J_{i j}-1\right)+\delta\left(J_{i j}-1\right)\right]+p_{1} \delta\left(J_{i j}\right) .
$$

In this way, the couplings $J_{i j}$ can be activating $(+1)$ or inhibitory $(-1)$, each with probability $\left(1-p_{1}\right) / 2$, or neutral $(0)$ with probability $p_{1}$. Furthermore, the linkages for every gene are chosen either at random among the whole Version Jan. 21, 2002...........Edited by : Typeset on 30 October $2018-2 \mathrm{~h} 18$ 
set of genes, with probability $p_{2}$, or only among the nearest neighbors, with probability $1-p_{2}$. By varying the parameter $p_{2}$ one can go from lattices where local interactions are the most important, to random nets where all ranges of interaction are present.

By analyzing the Hamming distance between two initial configurations, the authors show that there are three phases in the dynamics of the system: a frozen phase, a marginal phase and a chaotic phase, as shown in Fig. 6.2. The frozen and chaotic phases are as in the traditional $N-K$ model. The marginal phase is characterized by the fact that all the attractors are stable since a small change in the initial configuration neither vanishes nor grows. Also, in this phase both the number of different attractors and their lengths grow as a power of $N$. The above results were obtained for networks with connectivity $K=9$ and $N=625$. It is probable that the marginal phase is only a finite-size effect, vanishing for an infinite system. More work has to be done to explore the whole space of parameters of this model, but these preliminary results show that it is possible to obtain ordered and marginal behaviors even in the case of high connectivities by changing the type of interaction between the genes.

\subsection{Evolution}

In a traditional framework of the theory of evolution (Simon [1969; Dawkins [1989, 1986]), changes in the phenotype of organisms are accumulated gradually, yielding a gradual increase in complexity of form and function. Simple structures slowly assemble together to form more complex structures, which in turn assemble to build up even more elaborate systems, and so on. At every stage in the formation of a complex system (organism) out of simpler elements, many sub-systems are created, which represent temporal stable states along the way in the construction of the whole system. As T. D. Zawidzki has pointed out in Zawidzki [1998], from this point of view the problem of evolution of complex systems translates into a search problem. For the construction of a complex system, evolution searches in the space of the possible configurations leading to the whole system. If no stable intermediate configurations were found in this search, the construction of the whole system would be extremely improbable, since the number of possible configurations in which the parts can be arranged increases exponentially with the number of different parts. Evolution does not search in one step for the "final" configuration of a complex system. Instead, the search is carried out gradually, finding first intermediate stable configurations of sub-systems that are then assembled, giving rise to the whole complex system. Every sub-system solves a particular problem (or set of problems), facilitating the construction and functioning of the whole organism. Furthermore, once an evolutionary problem has been solved by primitive organisms, more complex species which evolve from them still

continue solving this problem in the same way. New species are faced with

Version Jan. 21, 2002...........Edited by : Typeset on 30 October $2018-2 \mathrm{~h} 18$ 
new problems, but still preserving the old solutions to the old problems (the genetic code, for example, was "invented" only once). According to this thesis, genomes of complex organisms are made up of functional modules of genes, each module encoding the solution of a given biological problem encountered by the species at some point through evolution.

The study of Boolean networks has suggested new mechanisms for evolutionary processes. Kauffman has stressed that many evolutionary changes involve reorganizing of the same genetic material rather than making it more complex (Kauffman [1993, 1995). In Kauffman's approach, genes are organized in complex genetic networks provided with some given dynamics. The "search" of evolution consists in searching for the most stable organization of genes, which in terms of the $N-K$ model means stable cycles. From this point of view, there is not an increasing complexification of the evolving system, but a inherent complex organizational dynamics which settles down in a finite number of stable attractors. The "role" of evolution then is to look for the more stable attractors which the system can fall into.

A Boolean network made up of $N$ genes has $2^{N}$ states, but the system organizes itself into a much smaller number of attractors. Depending on the parameter values being used $(K$ and $p)$, these attractors are stable or they are not. Stability is defined according to the response of the network to perturbations, which can be of three different kinds:

- changes in the states of a few genes by flipping the value of some randomly chosen ones;

- permanent changes in the linkages of some genes;

- permanent changes in the values of the evolution functions $f_{i}$ associated with some genes.

As we have already mentioned, only networks in the critical phase have the stability required to constitute evolvable systems, in that these networks are able to recover to most of the mutations described above. In the chaotic phase, the attractors are extremely unstable since any kind of perturbation would shift the system to another attractor. In the frozen phase, even though the majority of the genes are motionless, small changes in the linkages or in the evolution functions would make the system jump to a very different attractor if these damages are carried out on the relevant elements of the net. But networks operating in the critical phase show a very high homeostatic stability, which means that after some perturbation, such networks are very likely to fall again in the same attractor. Consequently, in this approach, evolution is also interpreted as a sort of search for gene networks possessing stable dynamics and not merely as a searching for stable sub-systems out of which more complicated systems can be built up. The fact that more complex living organisms have bigger amounts of genetic material, together with the fact that real genetic networks actually exhibit high homeostatic stability, suggest that evolutionary processes

Version Jan. 21, 2002 ...........Edited by : Typeset on 30 October $2018-2 \mathrm{~h} 18$ 
consist of both kinds of "searching," hierarchical-modular complexification and dynamical stability.

So far we have considered processes occurring at the level of genomes, but evolution also acts at the level of populations, making the organisms of a given species become better adapted to their environment. Living beings are subjected to all kinds of external fluctuations, and the survival of the species depends on the capability of its members to recover from those random perturbations. Evolutionary processes produce organisms with a high degree of homeostatic stability and of adaptability to the environment, even in the presence of external fluctuations. When thinking of evolutionary processes, one usually supposes that living organisms tend to exclude noise since stability is more conductive to functioning than chaos, and also that evolutionary processes are able to recognize and favor such stability. Nevertheless, Michael D. Stern has pointed out that none of these assumptions has been rigorously proven yet (Stern [1999). Furthermore, he has shown that, under certain circumstances, noise not only is not excluded from an adaptative system, but it is required for the adaptation of the system to the environment; without noise, such adaptation would not be possible.

In this work Stern considers a population of $M$ organisms, each of which is a $N-K$ model composed of $N=100$ elements. Each organism is in the critical phase ( $K=2$ and $p=0.5)$ and therefore, according to Kauffman, they are in the state of highest homeostatic stability. The linkages among the elements and the evolution rules $f_{i}$ are assigned in the usual way, but additionally, a noisy signal $\eta(t)$ is applied to $m$ randomly chosen elements of every organism. The noise is introduced through only one of the two inputs of each one of the $m$ elements. Suppose for example that $\sigma_{i_{1}}$ and $\sigma_{i_{2}}$ are the two inputs of $\sigma_{i}$. With no noise, the value of $\sigma_{i}$ at time $t+1$ would be given, as usual, by

$$
\sigma_{i}(t+1)=f_{i}\left(\sigma_{i_{1}}(t), \sigma_{i_{2}}(t)\right) .
$$

But if $\sigma_{i}$ is one of the $m$ elements to which the noisy signal $\eta(t)$ is being applied, then the value of $\sigma_{i}$ at time $t+1$ is given now by

$$
\sigma_{i}(t+1)=f_{i}\left(\sigma_{i_{1}}(t), \eta(t)\right) .
$$

Note that the noisy signal $\eta(t)$ is the same for the $m$ elements of every one of the $M$ organisms. This takes into account the fact that in a real population, every organism in the population is subjected to the same (noisy) environment.

The evolution of the population is now determined by an external criteria which has to be fulfilled. This is the phenotype of the population on which natural selection will be acting. In Stern's work, the phenotype to be selected is an integer time series, obtained by counting the number of positive states $(+1)$ occurring in a given subset of elements (output elements)

Version Jan. 21, 2002 ...........Edited by : Typeset on 30 October 2018 - 2h18 
in each organism. This time series is to fit a predefined time function $F(t)$ (the target function), and in each generation the organisms better adapted to $F(t)$ are selected.

Selection of the organisms is made as follows. At the beginning of each generation, every organism is replicated $R$ times allowing some mutations which consist mainly of randomly moving one of the input connections of a randomly chosen element, and randomly changing the Boolean function of a randomly chosen element. So, at the beginning of every generation the population actually consists of $R \times M$ elements. The system is then evolved during 100 time steps, after which the $M$ elements which best fit the external criteria $F(t)$ are selected, starting another generation.

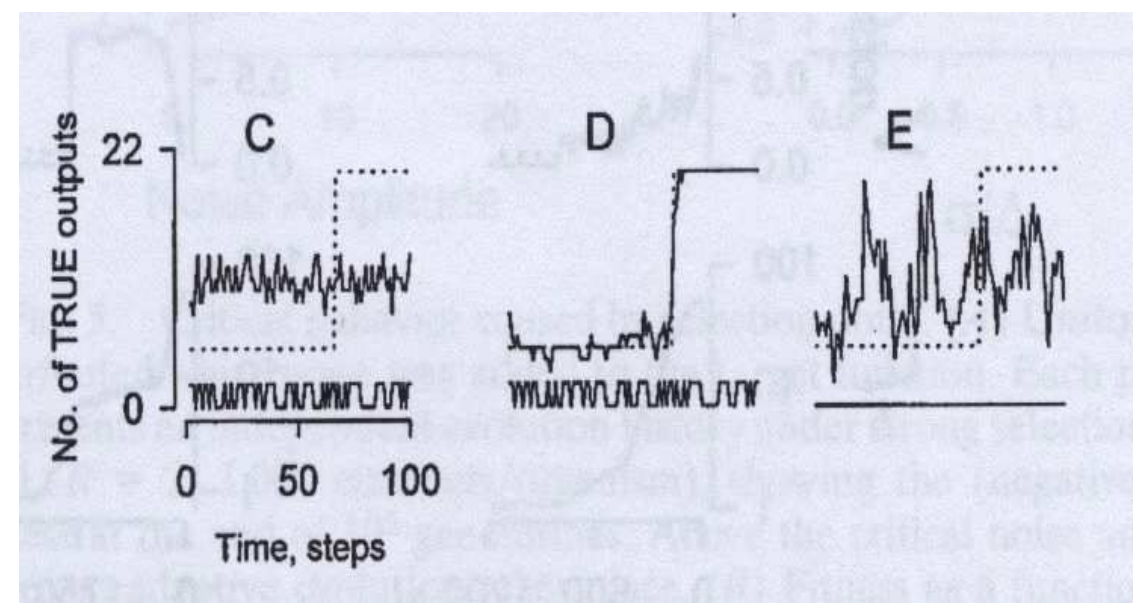

FIGURE 6.3. (C) Random output of the starting network (solid), the target function (dotted), and the fixed binary noise added to the system (lower) in Stern's model. (D) The evolved network generates a good approximation of the target function after 2500 generations. (E) The same network as in D but operating in the absence of noise fails completely in fitting the target function, indicating that the evolved network has been "imprinted" by the arbitrary noise sequence present during its evolution and requires it to function (taken from Stern [1999).

The results of the simulation are shown in Fig. 6.3, in which three output series are compared with the target function $F(t)$ (dotted line). Fig. 6.3 C shows the output signal of the initial generation, which has not yet passed through any selection process. In the presence of noise $\eta(t)$, after 2500 generations the target function is very well approximated by the output signal of the evolving network, as shown if Fig. 6.3D. However, in the absence of noise the same network fails completely to approximate the target function (Fig. 6.3 E). This surprising result implies that noisy perturbations may be essential for the adaptation of organisms to their environments. Stern has called this phenomenon noise imprinting in evolution, and claims that it 
"may be a prototype of a general form of symmetry breaking that leads to the evolution of structures that are suboptimal and unnecessarily dependent on fortuitous features of the environment".

\subsection{Social Systems}

It is always difficult to extrapolate the models and techniques of physics to the realm of social sciences. To begin with, it is not yet clear how to define a social system in a way that is suitable for the kind of analysis that physicists are used to. The complexity we see in human societies is of a quite different nature than the one present in physical systems, so one must be extremely cautious when talking about "complexity" and "chaos" in social sciences. Nonetheless, some parallels can be established between the complex behaviors of Boolean networks and human organizations.

Boolean networks were first introduced in social sciences by Schelling in 1971 (Shelling [1971). Following Klüver and Schmidt [1999], the main aspect in a social system is that social actors act according to certain social rules; the intended or unintended consequences of their actions generate other actions by the same or other actors and so forth. The result of such interactions is reflected in the dynamics of the system.

A key difficulty in the modeling of social networks is determining the quantities that convey enough information to describe a given system, and which have some degree of predictability. One must also identify the key parameters governing the behavior. Two kind of parameters can be distinguished: those which determine the topology of the system, namely, how individuals interact, and the ones which determine the social rules, i.e., the consequences of the interactions. To illustrate these two kind of parameters, we can focus our attention on the connectivity $K$ of Boolean networks and the weight parameter $p$ associated to the evolution functions $f_{i}$.

In ancient societies, characterized by very rigid dictatorial or monarchic regimes, the connectivity among people was very small since the majority of individuals were restricted to interact only with the few people in their communities. Mobility of individuals among different social classes was also minimal. Poor people were condemned to remain poor while rich people usually remained rich; social mobility, if any, was allow only from upper to lower classes. The course of the whole society depended largely on very few people (the King and his clique). In contrast, modern democratic societies are characterized by much bigger connectivities since individuals have a broader spectrum of interactions. In addition, social mobility is greater in democratic societies since, at least in principle, anybody can become president or actor or any other thing.

A binary network can be used for the modeling of a society of interacting individuals, in which poverty is represented by 1 while richness is represented by 0 . Of course, in real societies the connectivity among individuals varies from one individual to another, and so does the range of influence of

Version Jan. 21, 2002 ...........Edited by : Typeset on 30 October $2018-2 \mathrm{~h} 18$ 
their decisions; but it is clear that even if we assign the same connectivity to each member of the society, the out-degree will not be the same for all individuals ${ }^{12}$, and therefore the range of influence that each member of the society has on the other members varies among them.

With this setup, a rigid feudal society would be characterized by low values of the parameter $K$ and by evolution functions $f_{i}$ whose weight parameter $p$ is very low, reflecting the fact that the majority of the individuals are poor no matter what they do or whom they interact with. This kind of society presents a very simple dynamics in a frozen regime; no changes are expected nor unpredictable behavior. The dynamics is governed by a very reduced fraction of relevant elements, while the rest of the individuals remain in a frozen state with nearly zero mobility.

On the other hand, a perfect democratic society would be rather characterized by high connectivities and weighted functions $f_{i}$ whose parameter $p$ is close to 0.5 . In this situations, we have seen that the system exhibits chaotic behavior, with an apparently random dynamics. The society would never reach a stable state or a stable attractor, for in this regime the length of the cycles is extremely large.

If these results reflect some of the fundamental aspects behind the dynamics of social organizations, the conclusion would be that the complexity that we see in modern democratic societies is inherent to the democratic principles (parameter values) on which these societies have been constructed. Apparently, democracy and complexity are tied together, and it is a political decision whether or not it is worthwhile to sacrifice democracy in the interest of predictability. We should stress, though, that Boolean networks are far from accurate representations of a human society, so we should consider the previous results only as indicative trends and not as the matter of the facts.

\subsection{Neural Networks}

The first neural network model was introduced nearly 60 years ago by McCulloch and Pitts in an attempt to understand some of the cognitive processes of the brain (McCulloch and Pitts [1943). The topic of neural networks has since grown enormously, and presently it covers a great variety of fields, ranging from neurophysiology to computational algorithms for pattern recognition and non-parametric optimization processes Cheng and Titterington [1994]). Nonetheless, it is somehow accepted that the two major applications of neural networks consist in the understanding of real neural systems (the brain), and in the development of machine-learning algorithms (Farmer [1990]). In this section, we will address the subject from

\footnotetext{
${ }^{12}$ The out-degree of node $\sigma_{i}$ is the number of different nodes which are affected by $\sigma_{i}$, and can run from 0 up to $N$.
}

Version Jan. 21, 2002...........Edited by : Typeset on 30 October $2018-2 \mathrm{~h} 18$ 
a point of view based on statistical physics.

As in the $N-K$ model, a typical neural network consists of a set of $N$ nodes, each of which receives inputs from $K$ other nodes. The difference from the $N-K$ model is that in a neural network the dynamics are given by

$$
\sigma_{i}(t+1)=\operatorname{Sign}\left(\sum_{j=1}^{K} w_{i j} \sigma_{i_{j}}(t)+h\right)
$$

where the synapse weights $\left\{w_{i j}\right\}$ and the activation threshold $h$ are random variables that can adapt (in learning processes) or stay fixed (in recall or association processes). Note that with the above definitions, the nodes have the values $\sigma_{i}= \pm 1$.

K. E. Kürten has considered the case in which the synapse weights are independent random variables distributed according to a symmetric probability function $\mathrm{P}(w)$. By analyzing the Hamming distance, he has shown that this kind of neural network has behavior similar to that of the $\mathrm{N}$ $K$ model, in that the three phases (frozen, critical and chaotic) are also present (Kürten [1988a. []), depending on the connectivity $K$ and the dilution of the network (dilution is a measure of the amount of nodes for which the synapse weight is 0 ). The main result is that for low connectivity and dilution, the neural network and the $N-K$ model exhibit exactly the same dynamics in the Hamming distance.

Another important application of neural networks consists in investigating the tolerance to the influence of external noise in the dynamical organization of the network. Huepe and Aldana-González [2002] have analyzed

${ }^{13}$ There are other choices for the dynamics, such as

$$
\sigma_{i}(t+1)=\tanh \left(\sum_{j=1}^{K} w_{i j} \sigma_{i_{j}}(t)+h\right),
$$

which produces an output between -1 and +1 , or

$$
\sigma_{i}(t+1)=\left(1+\exp \left\{\sum_{j=1}^{K} w_{i j} \sigma_{i_{j}}(t)+h\right\}\right)^{-1}
$$

with an output between 0 and 1. Possible choices abound, depending on the particular task for which the network has been designed. We will focus on the particular class of neural networks obeying the dynamics given by equation 6.5 .

Version Jan. 21, 2002...........Edited by : Typeset on 30 October $2018-2 \mathrm{~h} 18$ 


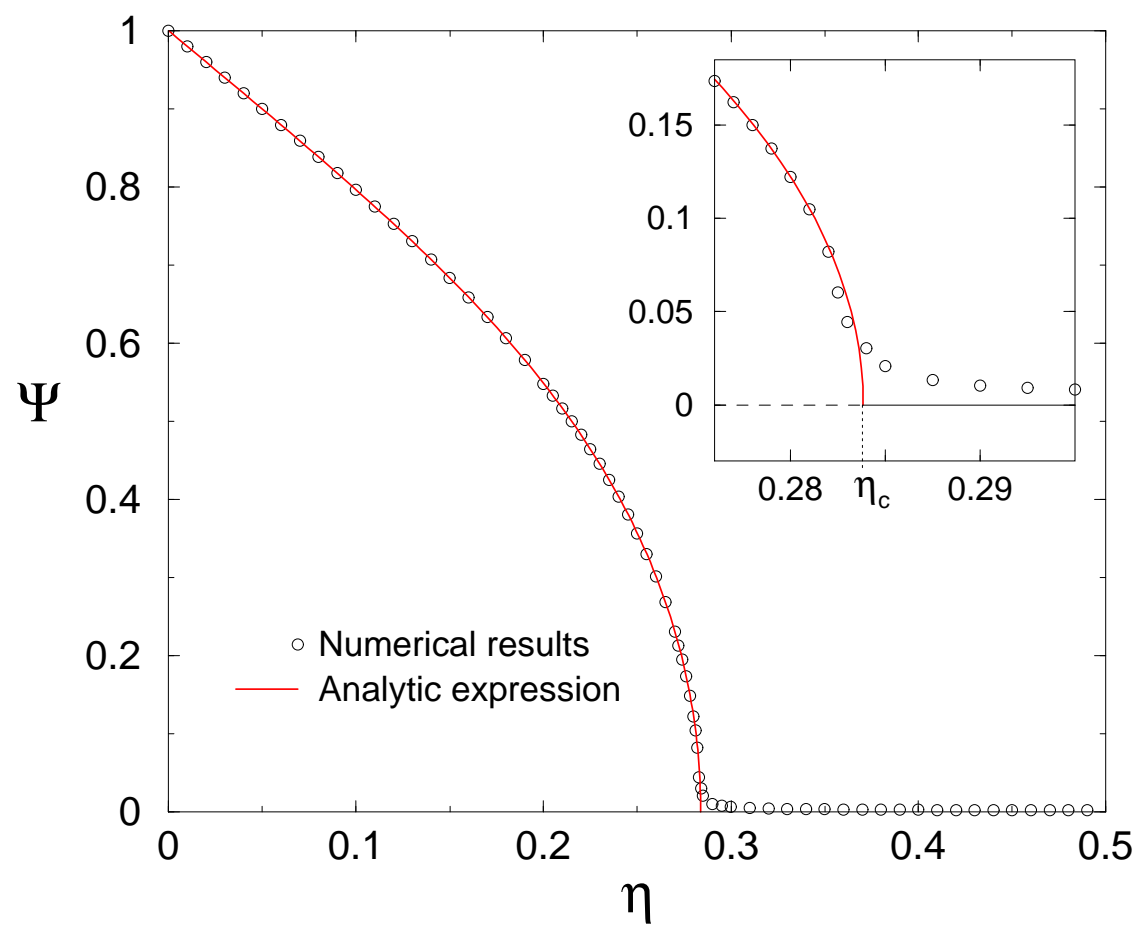

FIGURE 6.4. Bifurcation diagram of the order parameter $\Psi$ as a function of the noise intensity $\eta$, for the neural network model in (6.6). In the case shown, the network was composed of $N=10^{5}$ elements with connectivity $K=11$, and the synapse weights $w_{i j}$ were uniformly distributed between 0 and 1 . The inset shows the finite-size effects in the numerical results near the critical noise $\eta_{c} \simeq 0.2838$. (Taken from Huepe and Aldana-González [2002].)

a neural network whose dynamics is given by

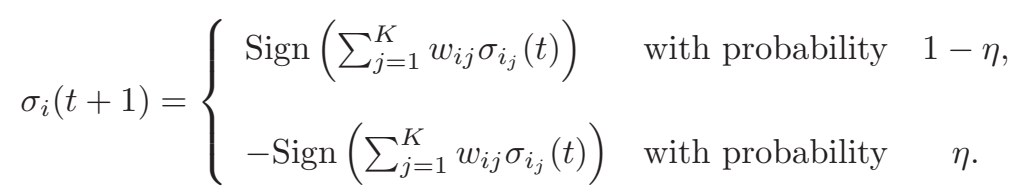

The dynamics can thus be changed from purely deterministic to purely random by varying the noise intensity $\eta$ between 0 and $1 / 2$. In the case $\eta=0$ the network will typically converge to an ordered state in which all the $\sigma_{i}$ acquire the same value. For other values of $\eta$, the instantaneous 
amount of order in the networks is determined by the parameter

$$
s(t)=\lim _{N \rightarrow \infty} \frac{1}{N} \sum_{i=1}^{N} \sigma_{i}(t),
$$

so that $|s(t)| \approx 1$ for an "ordered" system in which most elements take the same value, while $|s(t)| \approx 0$ for a "disordered" system in which the elements randomly take values +1 or -1 . The order parameter of the network is defined then as the time-independent quantity

$$
\Psi=\lim _{T \rightarrow \infty} \frac{1}{T} \int_{0}^{T}|s(t)| d t .
$$

Huepe and Aldana have shown that, under very general conditions, the neural network model given in (6.6) undergoes a dynamical second order phase transition, as it is illustrated in Fig. 6.4. More specifically, if the probability density $\mathrm{P}(w)$ of the synapse weights is a non-symmetric, but otherwise arbitrary function, and if the linkages of the network are chosen randomly, then there exists a critical value $\eta_{c}$ of the noise in the vicinity of which the order parameter behaves as

$$
\Psi= \begin{cases}C\left(\eta_{c}-\eta\right)^{1 / 2} & \text { for } \quad \eta<\eta_{c} \\ 0 & \text { for } \eta>\eta_{c}\end{cases}
$$

where $C$ and $\eta_{c}$ are constants whose values depend on the connectivity $K$ and on the particular form of $\mathrm{P}(w)$. It is worth mentioning that the dynamics of the network are exactly the same if both the linkages and the synapse weights $w_{i j}$ are time-independent, or if they are re-assigned at every time step. As far as we know, this is the first network model in which it has been shown that the quenched and annealed approaches coincide exactly.

Other models of particular interest are those in which the synapse weights $w_{i j}$ are in turn given by "sub-layers" of connections, such as in the LittleHopfield model (Hopfield [1982]; Little [1974]),

$$
w_{i j}=C_{i j} \sum_{\mu=1}^{p} \xi_{i}^{\mu} \xi_{j}^{\mu}
$$

where $\xi_{i}^{\mu}= \pm 1$ is the value of site $i$ in the sub-layer $\mu$, and the coefficients $C_{i j}$ are independent random variables distributed according to some given probability distribution $\mathrm{P}(C)$. These networks are important because by suitable changing the coefficients $C_{i j}$, the network can be either synchronized or "taught" some particular task (Hopfield [1999); Abarbanel, Rabinovich, Selverston, and Bazhenov [1996; Derrida, Gardner, and Zippelius [1987]).

Version Jan. 21, 2002...........Edited by : Typeset on 30 October $2018-2 \mathrm{~h} 18$ 


\section{Acknowledgements}

We would like to thank Xiaohui Qu for her valuable help in the elaboration of this article. This work was partially supported by the MRSEC Program of the NSF under Award Number 9808595, and by the NSF-DMR 0094569. We also thank the Santa Fe Institute of Complex Systems for partial support through the David and Lucile Packard Foundation Program in the Study of Robustness. M. Aldana also acknowledges CONACyT-México for a postdoctoral grant. 


\section{Bibliography}

Abarbanel, H. D. I., M. I. Rabinovich, A. Selverston, and M. V. Bazhenov [1996], Synchronization in Neural Networks, Physics-Uspeki 39, 337-362.

Albert, R. and A.-L. Barabási [2000], Dynamics of Complex Systems: Scaling Laws for the Period of Boolean Networks, Physical Review Letters 84, 5660-5663.

Albert, R. and A.-L. Barabási [2001], Statistical Mechanics of Complex Networks, LANL Data Base - http://arXiv.org/abs/cond-mat/0106096.

Alberts, B., D. Bray, J. Lewis, M. Raff, K. Roberts, and J. D. Watson [1994], Molecular Biology of the Cell, Third Edition. Garland Publishing, New York.

Andrecut, M. and M. K. Ali [2001], Chaos in a Simple Boolean Network, International Journal of Modern Physics B 15, 17-23.

Atlan, H., F. Fogelman-Soulie, J. Salomon, and G. Weisbuch [1981], Random Boolean Networks, Cybernetics and Systems 12, 103-121.

Bagley, R. J. and L. Glass [1996], Counting and Classifying Attractors in High Dimensional Dynamical Systems, Journal of Theoretical Biology 183, 269-284.

Baillie, C. F. and D. A. Johnston [1994], Damaging 2D Quantum Gravity, Physics Letters B 326, 51-56.

Bak, P., H. Flyvbjerg, and B. Lautrup [1992], Coevolution in a Rugged Fitness Landscape, Physical Review A 46, 6724-6730.

Bastolla, U. and G. Parisi [1996], Closing Probabilities in the Kauffman Model: an Annealed Computation, Physica D 98, 1-25.

Bastolla, U. and G. Parisi [1997], A Numerical Study of the Critical Line of Kauffman Networks, Journal of Theoretical Biology 187, 117-133.

Bastolla, U. and G. Parisi [1998a], The Modular Structure of Kauffman Networks, Physica D 115, 219-233.

Bastolla, U. and G. Parisi [1998b], Relevant Elements, Magnetization and Dynamical Properties in Kauffman Networks: a Numerical Study, Physica D 115, 203-218.

Version Jan. 21, 2002...........Edited by : Typeset on 30 October $2018-2 \mathrm{~h} 18$ 
Bhattacharjya, A. and S. Liang [1996a], Median Attractor and Transients in Random Boolean Nets, Physica D 95, 29-34.

Bhattacharjya, A. and S. Liang [1996b], Power-Law Distributions in Some Random Boolean Networks, Physical Review Letters 77, 1644-1647.

Bilke, S. and F. Sjunnesson [2001], Stability of the Kauffman Model, Physical Review E 65, 016129.

Bornholdt, S. [1998], Genetic Algorithm Dynamics on a Rugged Landscape, Physical Review E 57, 3853-3860.

Bornholdt, S. and T. Rohlf [2000], Topological Evolution of Dynamical Networks: Global Criticality From Local Dynamics, Physical Review Letters 84, 6114-6117.

Bornholdt, S. and K. Sneppen [1998], Neutral Mutations and Punctuated Equilibrium in Evolving Genetic Networks, Physical Review Letters 81, 236-239.

Bornholdt, S. and K. Sneppen [2000], Robustness as an Evolutionary Principle, Proc. Royal Soc. Lond. B 266, 2281-2286.

Bull, L. [1999], On the Baldwin Effect, Artificial Life 5, 241-246.

Burda, Z., J. Jurkiewicz, and H. Flyvbjerg [1990], Classification of Networks of Automata By Dynamic Mean-Field Theory, Journal of Physics A: Mathematical and General 23, 3073-3081.

Castagnoli, G. [1998], Merging Quantum Annealing Computation and Particle Statistics: A Prospect in the Search of Efficient Solutions to Intractable Problems, International Journal of Theoretical Physics 37, $457-462$.

Cheng, B. and D. M. Titterington [1994], Neural networks: a review from a statistical perspective, Statistical Science 9, 2-54.

Coppersmith, S. N., L. P. Kadanoff, and Z. Zhang [2001a], Reversible Boolean Networks I: Distribution of Cycle Lengths, Physica D 149, 1129 .

Coppersmith, S. N., L. P. Kadanoff, and Z. Zhang [2001b], Reversible Boolean Networks II: Phase Transitions, Oscilations and Local Structures., Physica D 157, 54-74.

Corsten, M. and P. Poole [1988], Initiation of Damage in the Kauffman Model, Journal of Statistical Physics 50, 461-463.

Dawkins, R. [1986], The Blind Watchmaker. W.W. Norton and Company, USA. 
Dawkins, R. [1989], The Selfish Gene. Oxford University Press, Oxford, second edition.

De Sales, J. A., M. L. Martins, and D. A. Stariolo [1997], Cellular Automata Model for Gene Networks, Physical Review E 55, 3262-3270.

Derrida, B. [1980], Random-Energy Model: Limit of a Family of Disordered Models, Physical Review Letters 45, 79-82.

Derrida, B. [1987a], Dynamical Phase Transitions in Non-Symmetric Spin Glasses, Journal of Physics A: Mathematical and General 20, L721$\mathrm{L} 725$.

Derrida, B. [1987b], Valleys and Overlaps in Kauffman Model, Philosophical Magazine B: Physics of Condensed Matter, Statistical Mechanics, Electronic, Optical and Magnetic Properties 56, 917-923.

Derrida, B. and D. Bessis [1988], Statistical Properties of Valleys in the Annealed Random Map Model, Journal of Physics A: Mathematical and General 21, L509-L515.

Derrida, B. and H. Flyvbjerg [1986], Multivalley Structure in Kauffman Model - Analogy With Spin-Glasses, Journal of Physics A: Mathematical and General 19, 1003-1008.

Derrida, B. and H. Flyvbjerg [1987a], Distribution of Local Magnetizations in Random Networks of Automata, Journal of Physics A: Mathematical and General 20, L1107-L1112.

Derrida, B. and H. Flyvbjerg [1987b], The Random Map Model: a Disordered Model With Deterministic Dynamics, Journal De Physique 48, 971-978.

Derrida, B., E. Gardner, and A. Zippelius [1987], An Exactly Solvable Asymmetric Neural Network Model, Europhysics Letters 4, 167-173.

Derrida, B. and Y. Pomeau [1986], Random Networks of Automata - a Simple Annealed Approximation, Europhysics Letters 1, 45-49.

Derrida, B. and D. Stauffer [1986], Phase-Transitions in Two-Dimensional Kauffman Cellular Automata, Europhysics Letters 2, 739-745.

Derrida, B. and G. Weisbuch [1986], Evolution of Overlaps Between Configurations in Random Boolean Networks, Journal De Physique 47, 1297 1303.

Domany, E. and W. Kinzel [1984], Equivalence of Cellular Automata to Ising Models and Directed Percolation, Physical Review Letters 53, 311314.

Version Jan. 21, 2002 ...........Edited by : Typeset on 30 October $2018-2 \mathrm{~h} 18$ 
Fambrough, D., K. Mcclure, A. Kazlauskas, and E. S. Lander [1999], Diverse Signaling Pathways Activated By Growth Factor Receptors Induce Broadly Overlapping, Rather That Independent, Sets of Genes, Cell 97, 727-741.

Farmer, J. D. [1990], A Roseta Stone for Connectionism, Physica D 42, 153-187.

Flyvbjerg, H. [1988], An Order Parameter for Networks of Automata, Journal of Physics A: Mathematical and General 21, L955-L960.

Flyvbjerg, H. [1989], Recent Results for Random Networks of Automata, Acta Physica Polonica B 20, 321-349.

Flyvbjerg, H. and N. J. Kjaer [1988], Exact Solution of Kauffman Model with Connectivity One, Journal of Physics A: Mathematical and General 21, 1695-1718.

Flyvbjerg, H. and B. Lautrup [1992], Evolution in a Rugged Fitness Landscape, Physical Review A 46, 6714-6723.

Fogelman-Soulie, F. [1984], Frustration and Stability in Random Boolean Networks, Discrete Applied Mathematic 9, 139-156.

Fogelman-Soulie, F. [1985], Parallel And Sequential Computation On Boolean Networks, Theor. Comp. Sci. 40, 275-300.

Genoud, T. and J.-P. Metraux [1999], Crosstalk in Plant Cell Signaling: Structure and Function of the Genetic Network, Trends in Plant Science 4, 503-507.

Glass, L. and C. Hill [1998], Ordered and Disordered Dynamics in Random Networks, Europhysics Letters 41, 599-604.

Golinelli, O. and B. Derrida [1989], Barrier Heights in the Kauffman Model, Journal De Physique 50, 1587-1601.

Griffiths, R. [1969], Nonanalytic Behavior Above the Critical Point in a Random Ising Ferromagnet, Physical Review Letters 23, 17-19.

Hansen, A. [1988a], A Connection Between the Percolation Transition and the Onset of Chaos In the Kauffman Model, Journal of Physics A: Mathematical and General 21, 2481-2486.

Hansen, A. [1988b], Percolation and Spreading of Damage in a Simplified Kauffman Model, Physica A 153, 47-56.

Harris, B. [1960], Probability Distributions Related to Random Mappings, Annals of Mathematical Statistics 31, 1045-1062.

Version Jan. 21, 2002 ...........Edited by : Typeset on 30 October 2018 - 2h18 
Herrmann, H. J. [1992], Simulation of Random Growth-Processes, Topics in Applied Physics 71, 93-120.

Hilhorst, H. J. and M. Nijmeijer [1987], On the Approach of the Stationary State in Kauffmans Random Boolean Network, Journal De Physique 48, 185-191.

Hopfield, J. J. [1982], Neural Networks and Physical Systems with Emergent Collective Computational Abilities, Proceedings of the National Academy of Sciences 79, 2554-2558.

Hopfield, J. J. [1999], Brain, Neural Networks and Computation, Reviews of Modern Physics 71, S431-S437.

Huang, S. and D. E. Ingber [2000], Shape-Dependent Control of Cell Growth, Differentiation, and Apoptosis: Switching Between Attractors in Cell Regulatory Networks, Experimental Cell Research 261, 91-103.

Huepe, C. and M. Aldana-González [2002], Dynamical Phase Transition in a Neural Network Model with Noise: An Exact Solution, to appear in the Journal of Statistical Physics, 108 (3/4).

Ito, K. and Y.-P. Gunji [1994], Self-Organization of Living Systems Towards Criticality at the Edge of Chaos, Biosystems 33, 17-24.

Jan, N. [1988], Multifractality and the Kauffman Model, Journal of Physics A: Mathematical and General 21, L899-L902.

Kadanoff, L. P. [2000], Statistical Physics: Statics Dynamics and Renormalization. World Scientific, Singapore.

Kauffman, S. [1984], Emergent Properties in Random Complex Automata, Physica D 10, 145-156.

Kauffman, S. A. [1969], Metabolic Stability and Epigenesis in Randomly Constructed Nets, Journal of Theoretical Biology 22, 437-467.

Kauffman, S. A. [1974], The Large Scale Structure and Dynamics of Genetic Control Circuits: an Ensemble Approach, Journal of Theoretical Biology 44, 167-190.

Kauffman, S. A. [1990], Requirements for Evolvability in Complex Systems - Orderly Dynamics and Frozen Components, Physica D 42, 135-152.

Kauffman, S. A. [1993], The Origins of Order: Self-Organization and Selection in Evolution. Oxford University Press, Oxford.

Kauffman, S. A. [1995], At Home in the Universe: the Search for Laws of Self-Organization and Complexity. Oxford University Press, Oxford.

Version Jan. 21, 2002 ...........Edited by : Typeset on 30 October 2018 - 2h18 
Kauffman, S. A. and W. G. Macready [1995], Search Strategies for Applied Molecular Evolution, Journal of Theoretical Biology 173, 427-440.

Kauffman, S. A. and E. D. Weinberger [1989], The NK Model of Rugged Fitness Landscapes and Its Application To Maturation of the Immune Response, Journal of Theoretical Biology 141, 211-245.

Kaufman, J. H., D. Brodbeck, and O. M. Melroy [1998], Critical Biodiversity, Conservation Biology 12, 521-532.

Kirillova, O. V. [1999], Influence of a Structure on Systems Dynamics on Example of Boolean Networks, International Journal of Modern Physics C 10, 1247-1260.

Klüver, J. and J. Schmidt [1999], Control Parameters in Boolean Networks and Cellular Automata Revisited from a Logical and Sociological Point of View, Complexity 5, 45-52.

Krapivsky, P. L., S. Redner, and F. Leyvraz [2000], Connectivity of Growing Random Networks, Physical Review Letters 85, 4629-4632.

Kulakowski, K. [1995], Relaxation and Limit-Cycles in a Global Version of the Quenched Kauffman Model, Physica A 216, 120-127.

Kürten, K. E. [1988a], Correspondence Between Neural Threshold Networks and Kauffman Boolean Cellular Automata, Journal of Physics A: Mathematical and General 21, L615-L619.

Kürten, K. E. [1988b], Critical Phenomena in Model Neural Netwoks, Physics Letters A 129, 157-160.

Kürten, K. E. and H. Beer [1997], Inhomogeneous Kauffman Models at the Borderline Between Order and Chaos, Journal of Statistical Physics 87, 929-935.

Lam, P. M. [1988], A Percolation Approach to the Kauffman Model, Journal of Statistical Physics 50, 1263-1269.

Langton, C. G. [1990], Computations at the Edge of Chaos: Phase Transitions and Emergent Computation, Physica D 42, 12-37.

Lee, C.-Y. and S. K. Han [1998], Evolutionary Optimization Algorithm By Entropic Sampling, Physical Review E 57, 3611-3617.

Levitan, B. and S. Kauffman [1995], Adaptive Walks With Noisy Fitness Measurements, Molecular Diversity 1, 53-68.

Little, W. A. [1974], The Existence of Persistent States in the Brain, Mathematical Bioscience 19, 101-120.

Version Jan. 21, 2002 ...........Edited by : Typeset on 30 October $2018-2 \mathrm{~h} 18$ 
Luczak, T. and J. E. Cohen [1991], Stability of Vertices in Random Boolean Cellular Automata, Random Structures and Algorithms 2, 327-334. reference from Lynch.

Luque, B. and R. V. Solé [1997a], Controlling Chaos in Random Boolean Networks, Europhysics Letters 37, 597-602.

Luque, B. and R. V. Solé [1997b], Phase Transitions in Random Networks: Simple Analytic Determination of Critical Points, Physical Review E 55, $257-260$.

Luque, B. and R. V. Solé [1998], Stable Core and Chaos Control in Random Boolean Networks, Journal of Physics A: Mathematical and General 31, 1533-1537.

Luque, B. and R. V. Solé [2000], Lyapunov Exponents in Random Boolean Networks, Physica A 284, 33-45.

Lynch, J. F. [1993a], Antichaos in a Class of Random Boolean CellularAutomata, Physica D 69, 201-208.

Lynch, J. F. [1993b], A Criterion for Stability in Random Boolean CellularAutomata, Los Alamos Data Base http://arXiv.org/abs/adaporg/9305001.

Lynch, J. F. [1995], On the Threshold of Chaos in Random Boolean Cellular-Automata, Random Structures and Algorithms 6, 239-260.

Ma, S. [1976], Modern Theory of Critical Phenomena. Benjamin, Reading $\mathrm{Pa}$.

Macisaac, A. B., D. L. Hunter, M. J. Corsten, and N. Jan [1991], Determinism and Thermodynamics - Ising Cellular Automata, Physical Review A 43, 3190-319.

Manrubia, S. C. and A. S. Mikhailov [1999], Mutual Synchronization and Clustering in Randomly Coupled Chaotic Dynamical Networks, Physical Review E 60, 1579-1589.

McCulloch, W. S. and W. Pitts [1943], A Logical Calculus of Ideas Immanent in Nervous Activity, Bulletin of Mathematical Biophysics 5, 115133.

Mestl, T., R. J. Bagley, and L. Glass [1997], Common Chaos in Arbitrarily Complex Feedback Networks, Physicl Review Letters 79, 653-656.

Metropolis, N. and S. Ulam [1953], A Property of Randomness of an Arithmetical Function, American Mathematical Monthly 60, 252-253.

Version Jan. 21, 2002 ...........Edited by : Typeset on 30 October 2018 - 2h18 
Mezard, M., G. Parisi, and M. A. Virasoro [1987], Spin Glass Theory and Beyond. World Scientific, Singapore.

Miranda, E. N. and N. Parga [1988], Ultrametricity in the Kauffman Model - a Numerical Test, Journal of Physics A: Mathematical and General 21, L357-L361.

Miranda, E. N. and N. Parga [1989], Noise Effects in the Kauffman Model, Europhysics Letter 10, 293-298.

Nirei, M. [1999], Critical Fluctuations in a Random Network Model, Physica A 269, 16-23.

Obukhov, S. P. and D. Stauffer [1989], Upper Critical Dimension of Kauffman Cellular Automata, Journal of Physics A: Mathematical and General 22, 1715-1718.

Ohta, T. [1997a], The Meaning of Near-Neutrality at Coding and NonCoding Regions, Gene 205, 261-267.

Ohta, T. [1997b], Role of Random Genetic Drift in the Evolution of Interactive Systems, Journal of Molecular Evolution 44, S9-S14.

Ohta, T. [1998], Evolution By Nearly Neutral Mutations, Genetica 103, $83-90$.

Owezarek, A., A. Rechnitzer, and A. J. Guttmann [1997], On the Hulls of Directed Percolation Clusters, Journal of Physics A: Mathematical and General 30, 6679-6691.

Petters, D. [1997], Patch Algorithms in Spin Glasses, International Journal of Modern Physics C 8, 595-600.

Preisler, H. D. and S. Kauffman [1999], A Proposal Regarding the Mechanism Which Underlies Lineage Choice During Hematopoietic Differentiation, Leukemia Research 23, 685-694.

Qu, X., L. Kadanoff, and M. Aldana [2002], Numerical and Theoretical Studies of Noise Effects in Kauffman Model, Draft paper, unpublished.

Randeria, M., J. Sethna, and R. Palmer [1985], Low-Frequency Relaxation in Ising Spin-Glasses, Physical Review Letters 54, 1321-1324.

Rosser, J. B. and L. Schoenfeld [1962], Approximate Formulas for some Functions of Prime Numbers, Illinois Journal of Mathematics 6, 64-94.

Sakai, K. and Y. Miyashita [1991], Neural Organization for the Long-TermMemory of Paired Associates, Nature 354, 152-155.

Version Jan. 21, 2002...........Edited by : Typeset on 30 October $2018-2 \mathrm{~h} 18$ 
Serra, R. and M. Villani [1997], Modelling Bacterial Degradation of Organic Compounds With Genetic Networks, Journal of Theoretical Biology 189, 107-119.

Shelling, T. C. [1971], Dynamic Models of Segregation, Journal of Mathematical Sociology 1, 143-186.

Sherrington, D. and K. Y. M. Wong [1989], Random Boolean Networks for Autoassociative Memory, Physics Reports: Review Section of Physics Letters 184, 293-299.

Sherrington, D. and S. Kirkpatrick [1975], Solvable Model of a Spin-Glass, Physical Review Letters 35, 1792-1796.

Sibani, P. and A. Pedersen [1999], Evolution Dynamics in Terraced NK Landscapes, Europhysics Letters 48, 346-352.

Simon, H. A. [1969], The Sciences of the Artificial. The MIT Press, Cambridge, MA.

Solov, D., A. Burnetas, and M.-C. Tsai [1999], Understanding and Attenuating the Complexity Catastrophe In Kauffman's NK Model of Genome Evolution, Complexity 5, 53-66.

Solow, D., A. Burnetas, T. Roeder, and N. S. Greenspan [1999], Evolutionary Consequences of Selected Locus-Specific Variations In Epistasis and Fitness Contribution in Kauffman's NK Model, Journal of Theoretical Biology 196, 181-196.

Somogyi, R. and C. A. Sniegoski [1996], Modeling the Complexity of Genetic Networks: Understanding Multigenetic and Pleiotropic Regulation, Complexity 1, 45-63.

Somogyvári, Z. and S. Payrits [2000], Length of State Cycles of Random Boolean Networks: an Analytic Study, Journal of Physics A: Mathematical and General 33, 6699-6706.

Stadler, P. F. and R. Happel [1999], Random Field Models for Fitness Landscapes, Journal of Mathematical Biology 38, 435-478.

Stauffer, D. [1985], Introduction to Percolation Theory. Taylor and Francis, London.

Stauffer, D. [1994], Evolution By Damage Spreading in Kauffman Model, Journal of Statistical Physics 74, 1293-1299.

Stauffer, D. [1987a], On Forcing Functions in Kauffman Random Boolean Networks, Journal of Statistical Physics 46, 789-794.

Version Jan. 21, 2002 ...........Edited by : Typeset on 30 October 2018 - 2h18 
Stauffer, D. [1987b], Random Boolean Networks - Analogy With Percolation, Philosophical Magazine B: Physics of Condensed Matter, Statistical Mechanics, Electronic, Optical and Magnetic Properties 56, 901-916.

Stauffer, D. [1988], Percolation Thresholds in Square-Lattice Kauffman Model, Journal of Theoretical Biolog 135, 255-261.

Stauffer, D. [1989], Hunting for the Fractal Dimension of the Kauffman Model, Physica D 38, 341-344.

Stauffer, D. [1991], Computer Simulations of Cellular Automata, Journal of Physics A: Mathematical and General 24, 909-927.

Stern, M. D. [1999], Emergence of Homeostasis and Noise Imprinting in an Evolution Model, Proceedings of the National Academy of Sciences of the U.S.A. 96, 10746-10751.

Stölzle, S. [1988], Universality Two-Dimensional Kauffman Model for Parallel and Sequential Updating, Journal of Statistical Physics 53, 995-1004.

Strogatz, S. H. [2001], Exploring Complex Networks, Nature 410, 268-276.

Thieffry, D. and D. Romero [1999], The Modularity of Biological Regulatory Networks, Biosystems 50, 49-59.

Toffoli, T. and N. H. Margolus [1990], Invertible Cellular Automata: a Review, Physica D 45, 229-253.

Volkert, L. G. and M. Conrad [1998], The Role of Weak Interactions in Biological Systems: the Dual Dynamics Model, Journal of Theoretical Biology 193, 287-306.

Waelbroeck, H. and F. Zertuche [1999], Discrete Chaos, Journal of Physics A: Mathematical and General 32, 175-189.

Wang, L., E. E. Pichler, and J. Ross [1990], Oscillations and Chaos in Neural Networks - an Exactly Solvable Model, Proceedings of the National Academy of Sciences of the United States of America 87, 9467-9471.

Weinberger, E. D. [1991], Local Properties of Kauffman NK Model - a Tunably Rugged Energy Landscape, Physical Review A 44, 6399-6413.

Weisbuch, G. and D. Stauffer [1987], Phase Transitions in Cellular Random Boolean Networks, Jounal De Physique 48, 11-18.

Wilke, C. O., C. Ronnenwinkel, and T. Martinetz [2001], Dynamic Fitness Landscapes in Molecular Evolution, Physics Reports 349, 395-446.

Wolfram, S. [1983], Statistical Mechanics of Cellular Automata, Reviews of Modern Physics 55, 601-644.

Version Jan. 21, 2002...........Edited by : Typeset on 30 October $2018-2 \mathrm{~h} 18$ 
Wuensche, A. [1999], Discrete Dynamical Networks and their Attractor Basins, Complexity International 6, http://www.csu.edu.au/ci/idxvolume.html.

Zawidzki, T. W. [1998], Competing Models of Stability in Complex, Evolving Systems: Kauffman Vs. Simon, Biology and Philosophy 13, 541-554.

Zoli, M., D. Guidolin, K. Fuxe, and L. F. Agnati [1996], The Receptor Mosaic Hypothesis of the Engram: Possible Relevance of Boolean Network Modeling, International Journal of Neural Systems 7, 363-368.

Version Jan. 21, 2002...........Edited by : Typeset on 30 October $2018-2 \mathrm{~h} 18$ 